Pacific

Journal of

Mathematics

ON DEGENERATE SECANT VARIETIES WHOSE GAUSS MAPS HAVE THE LARGEST IMAGES

Masahiro OHNo

Volume $187 \quad$ No. 1

January 1999 


\title{
ON DEGENERATE SECANT VARIETIES WHOSE GAUSS MAPS HAVE THE LARGEST IMAGES
}

\author{
MASAHIRO OHNO
}

\begin{abstract}
We propose a class of projective manifolds with degenerate secant varieties, which class is wider than that of Scorza varieties, and study some properties of this class of manifolds. For example, we show that there is a strong restriction on dimensions of manifolds in this class. We also give classifications of such manifolds of low dimensions.
\end{abstract}

\section{Introduction.}

Let $X$ be an $n$-dimensional nondegenerate (i.e., not contained in a hyperplane) projective manifold in $\mathbf{P}^{N}$ over an algebraically closed field $k$ of characteristic 0. Let Sec $X$ denote the secant variety of $X$ in $\mathbf{P}^{N}$. It is well known that $\operatorname{dim} \operatorname{Sec} X \leq \min \{2 n+1, N\}$. If $\operatorname{dim} \operatorname{Sec} X<\min \{2 n+1, N\}$, $\operatorname{Sec} X$ is said to be degenerate (see $[\mathbf{L}])$. If $\operatorname{Sec} X$ is degenerate, we define the secant defect of $X$ to be the integer $\delta=2 n+1-\operatorname{dim} \operatorname{Sec} X$ (see $[\mathbf{L}-\mathbf{V}, \S 1 \mathrm{f}]$ or $[\mathbf{Z}$, Chap. 5, §1]). The Linear Normality Theorem ([Z, Chap. 2, Corollary 2.17]) implies that if $\operatorname{Sec} X$ is degenerate then $\operatorname{dim} \operatorname{Sec} X$ has a lower bound, that is, $\operatorname{dim} \operatorname{Sec} X \geq(3 n+2) / 2$. If equality holds, $X$ is called a Severi variety. Severi varieties were classified finally by F.L. Zak into only four manifolds up to projective equivalence ([Z, Chap. 4, Theorem 4.7]). After Zak's complete classification of Severi varieties, R. Lazarsfeld and A. Van de Ven proposed the following problem in $[\mathbf{L}-\mathbf{V}, \S 1 \mathrm{f}]$, observing that the secant defects of Severi varieties are less than or equal to eight.

Problem. Do there exist smooth projective varieties with arbitrarily large secant defect?

Zak also generalized the class of Severi varieties to a class of manifolds, named Scorza varieties, and classified Scorza varieties [Z, Chap. 6]. However the secant defects of Scorza varieties are also less than or equal to eight, and it is not yet known whether there exists a manifold with $\delta \geq 9$ (see Examples 1-9 in Section 1 and [L, Example 2.3]). In this article, we propose a new class of projective manifolds with degenerate secant varieties, which class is wider than the class of Scorza varieties, and investigate some properties of this class of manifolds. In particular we show that there is a strong restriction on dimension of the manifold $X$ in this class with large $\delta$, and we give a classification of such manifolds of low dimension. 
Suppose that $\operatorname{Sec} X$ is degenerate. Let $\varepsilon=2 \operatorname{dim} \operatorname{Sec} X-3 n-2$. Then $\varepsilon \geq 0$ and $X$ is a Severi variety if and only if $\varepsilon=0$. Let $\operatorname{Sm}(\operatorname{Sec} X)$ denote the smooth locus of $\operatorname{Sec} X$, and let $\gamma: \operatorname{Sm}(\operatorname{Sec} X) \rightarrow G\left(\operatorname{dim} \operatorname{Sec} X, \mathbf{P}^{N}\right)$ be the Gauss map $u \mapsto T_{u} \operatorname{Sec} X$ of $\operatorname{Sm}(\operatorname{Sec} X)$. We have the following proposition concerning the dimension of the image of $\gamma$.

Proposition 0.1. $\operatorname{dim} \operatorname{Im}(\gamma)=2(\operatorname{dim} \operatorname{Sec} X-n-1-c)$ for some integer $c$ $(0 \leq c \leq \varepsilon)$.

For a Severi variety $X$, the invariant $c$ in Proposition 0.1 is obviously zero, and not only Severi varieties but also Scorza varieties satisfy the condition $c=0$ (see $[\mathbf{Z}$, Chap. 6, (1.4.11)]). Moreover the condition $c=0$ is satisfied by other manifolds such as general hyperplane sections of any of Scorza varieties (see Examples 1-4 and 7 in Section 1). Furthermore, to the best of my knowledge, all examples of $c>0$ are constructed from those of $c=0$ (see Examples 8 and 9 Section 1). Thus we study projective manifolds with degenerate secant varieties whose Gauss maps have the largest images, i.e., $c=0$, in this article.

The main results of this article are the following.

Theorem 0.2. Suppose that $\operatorname{Sec} X$ is degenerate and that $\operatorname{dim} \operatorname{Im}(\gamma)=$ $2(\operatorname{dim} \operatorname{Sec} X-n-1)$. Then

1) $X$ is rationally connected.

2) If $\operatorname{dim} \operatorname{Sec} X \leq(2 n-2)$, then $\operatorname{dim} \operatorname{Sec} X \equiv n+1(\bmod 2)$, i.e., $n+\varepsilon \equiv 0$ $(\bmod 4)$, and $X$ is a Fano manifold with $K_{X} \cong \mathcal{O}_{X}((-3 n+\varepsilon) / 4)$.

3) If $\operatorname{dim} \operatorname{Sec} X \leq(2 n-4)$, then $\operatorname{dim} \operatorname{Sec} X \equiv n+1(\bmod 4)$.

4) The possible values of $n$ are

(4-1) a) $4,6,10,14$, or b) $2^{m}-2(m \geq 7), 2^{m} \cdot 3-2(m \geq 5)$ if $\varepsilon=2$;

(4-2) a) $5,7,9,13$, or b) $21,2^{m}-3(m \geq 7), 2^{m} \cdot 3-3(m \geq 5)$ if $\varepsilon=3$;

(4-3) a) $6,8,12$, or b) $20,28,2^{m}-4(m \geq 7), 2^{m} \cdot 3-4(m \geq 5)$ if $\varepsilon=4$;

(4-4) a) $7,9,11$, or b) $19,27,2^{m}-5(m \geq 7), 2^{m} \cdot 3-5(m \geq 5)$ if $\varepsilon=5$.

This theorem can be seen as a generalization of the famous result that if $\varepsilon=0$, i.e., $X$ is a Severi variety then $X$ is a Fano manifold and the possible values of $n$ are 2, 4, 8 and 16. In [Oh, Theorem 0.2], it is shown that if $\varepsilon=1$ and $\operatorname{dim} \operatorname{Im}(\gamma)=2(\operatorname{dim} \operatorname{Sec} X-n-1)$ then the possible values of $n$ are 3,5 , $7,15,2^{m}-1(m \geq 7)$, or $2^{m} \cdot 3-1(m \geq 5)$. However it is unknown whether there exists a manifold $X$ of $\operatorname{dim} \operatorname{Sec} X \leq 2 n-4$ and $n \geq 17$. Note also that all known examples satisfying the condition $\operatorname{dim} \operatorname{Im}(\gamma)=2(\operatorname{dim} \operatorname{Sec} X-n-1)$ are not only rationally connected but also Fano.

Theorem 0.3. Suppose that $\operatorname{Sec} X$ is degenerate and of dimension $2 n-1$ and that the image of the Gauss map $\gamma$ has dimension $2(n-2)$.

If $n=6$, then $\left(X, \mathcal{O}_{X}(1)\right)$ is one of the following.

1) $\left(\mathbf{P}^{l} \times \mathbf{P}^{6-l}, \mathcal{O}(1) \otimes \mathcal{O}(1)\right)(l=2,3)$;

2) $X \subset \mathbf{P}^{N}$ is a linear section of $G\left(1, \mathbf{P}^{5}\right)$ embedded in $\mathbf{P}^{14}$ via the Plücker 
embedding a section which is cut out by codimension 2 linear subspace of $\mathbf{P}^{14}$.

If Sec $X$ is degenerate and of dimension $2 n-1$, then $n \geq 4$ by the Linear Normality Theorem. If $n=4$, then $\left(X, \mathcal{O}_{X}(1)\right)$ is the 4-dimensional Severi variety $\left(\mathbf{P}^{2} \times \mathbf{P}^{2}, \mathcal{O}(1) \otimes \mathcal{O}(1)\right)$. In [OH, Theorem 0.3], it is shown that if $n=5$ and $\operatorname{dim} \operatorname{Im}(\gamma)=6$ then $\left(X, \mathcal{O}_{X}(1)\right)$ is isomorphic to $\left(\mathbf{P}^{2} \times \mathbf{P}^{3}, \mathcal{O}(1) \otimes\right.$ $\mathcal{O}(1))$.

Theorem 0.4. Suppose that $\operatorname{Sec} X$ is degenerate and of dimension $2 n$ and that $\operatorname{dim} \operatorname{Im}(\gamma)=2(n-1)$.

If $n=4$, then $\left(X, \mathcal{O}_{X}(1)\right)$ is one of the following.

1) $\left(\mathbf{P}_{\mathbf{P}^{l}}(\mathcal{E}), H(\mathcal{E})\right)$, where $\mathcal{E}=\mathcal{O}(1)^{\oplus(4-l)} \oplus \mathcal{O}(2)(l=2,3,4)$;

2) $\left(\mathbf{P}_{\mathbf{P}^{2}}(\mathcal{E}), H(\mathcal{E})\right)$, where $\mathcal{E}=\mathcal{O}(1) \oplus T_{\mathbf{P}^{2}}$.

If $n=5$, then $\left(X, \mathcal{O}_{X}(1)\right)$ is one of the following.

1) $\left(\mathbf{P}_{\mathbf{P}^{l}}(\mathcal{E}), H(\mathcal{E})\right)$, where $\mathcal{E}=\mathcal{O}(1)^{\oplus(5-l)} \oplus \mathcal{O}(2)(l=2,3,4,5)$;

2) $\left(\mathbf{P}_{\mathbf{P}^{l}}(\mathcal{E}), H(\mathcal{E})\right)$, where $\mathcal{E}=\mathcal{O}(1)^{\oplus(6-2 l)} \oplus T_{\mathbf{P}^{l}}(l=2,3)$;

3) $X \subset \mathbf{P}^{N}$ is a linear section of $G\left(1, \mathbf{P}^{5}\right) \subset \mathbf{P}^{14}$ a section cut out by codimension 3 linear subspace of $\mathbf{P}^{14}$;

4) $\left(\Sigma_{10}, \mathcal{O}(1)\right)$, where $\Sigma_{10}$ is the adjoint manifold of the simple algebraic group of exceptional type $G_{2}$ and $\mathcal{O}(1)$ is the fundamental line bundle on it. (In other words $\Sigma_{10}$ is the 5-dimensional Mukai manifold of genus 10 $([\mathrm{Mu}])$.

If Sec $X$ is degenerate and of dimension $2 n$, then $n \geq 2$ by the Linear Normality Theorem. If $n=2$, then F. Severi determined $\left(X, \mathcal{O}_{X}(1)\right)$ to be $\left(\mathbf{P}^{2}, \mathcal{O}(2)\right)$. If $n=3$, then T. Fujita $([\mathbf{F}$, Theorem (2.1)]) showed that $\left(X, \mathcal{O}_{X}(1)\right)$ is one of the following: $\left(\mathbf{P}_{\mathbf{P}^{l}}\left(\mathcal{O}(1)^{\oplus 3-l} \oplus \mathcal{O}(2)\right), H(\mathcal{O}(1) \oplus \mathcal{O}(2))\right)$ where $(l=2,3)$, or $\left(\mathbf{P}\left(T_{\mathbf{P}^{2}}\right), H\left(T_{\mathbf{P}^{2}}\right)\right)$.

The contents of this article are as follows. In Section 1, we collect examples and basic facts concerning degenerate secant varieties and give a proof of Proposition 0.1. In Section 2, we investigate projective manifolds with degenerate secant varieties whose Gauss maps have the largest images and show Theorem 0.2. In Section 3, we study projective manifolds with degenerate secant varieties of dimension $2 n-1$ and give a proof of Theorem 0.3. Finally in Section 4 we investigate the case that $\operatorname{dim} \operatorname{Sec} X=2 n$ and we prove Theorem 0.4.

Acknowledgements. The author expresses gratitude to Professors Takao Fujita and Hajime Kaji for their kind advice and encouragement, and to the referee for his valuable suggestions. 


\section{Notation and conventions.}

We work over an algebraically closed field $k$ of characteristic 0 . We follow the notation and terminology of $[\mathbf{H}]$. We use the word manifold to mean a smooth variety. For a manifold $X$, we denote by $K_{X}$ the canonical divisor of $X$ and by $\kappa(X)$ the Kodaira dimension of $X$. We use the word point to mean a closed point and the word line to mean a smooth rational curve of degree 1 . By a secant line of a subvariety $X$ of $\mathbf{P}^{N}$, we mean a line joining two distinct points of $X$. The embedded tangent space $T_{x} X$ of a subvariety $X$ of $\mathbf{P}^{N}$ at a point $x \in X$ is the unique linear subspace $L \subseteq \mathbf{P}^{N}$ such that $x \in L$ and there is an equality of Zariski tangent space $T(X)_{x}=T(L)_{x}$ as linear subspace of $T\left(\mathbf{P}^{N}\right)_{x}$. For a closed subvariety $X$ of $\mathbf{P}^{N}$, we call the secant variety of $X$ the closure of the union of all secant lines and denote it by $\operatorname{Sec} X$, and we call the tangent variety of $X$ the union of all embedded tangent spaces and denote it by $\operatorname{Tan} X$. We denote by $G\left(r, \mathbf{P}^{N}\right)$ the Grassmannian of $r$-planes in $\mathbf{P}^{N}$. Given two distinct points $x, y$ on $\mathbf{P}^{N}$, let $x * y$ denote the line joining them. For subsets $X, Y$ of $\mathbf{P}^{N}$, let $X * Y$ be the closure of the union of all lines $x * y$ joining two distinct points $x \in X$ and $y \in Y$. We use the words "locally free sheaf" and "vector bundle" interchangeably if no confusion seems likely to result. For a vector bundle $E$ of rank $e+1$ on a variety $X$, we define the $i$-th Segre class $s_{i}(E)$ of $E$ by the formula $s_{i}(E) \cap \alpha=p_{*}\left(c_{1}\left(\mathcal{O}_{\mathbf{P}\left(E^{\vee}\right)}(1)\right)^{e+i} \cap p^{*} \alpha\right)$ where $\alpha$ is a $k$-dimensional cycle modulo rational equivalence and $p: \mathbf{P}(E) \rightarrow X$ is the projection. We also define the total Segre class $s(E)$ to be $1+s_{1}(E)+s_{2}(E)+\cdots$. The total Chern class $c(E)=1+c_{1}(E)+c_{2}(E)+\cdots$ is defined by the formula $c(E) s(E)=1$. These definitions of $s_{i}(E)$ and $c_{i}(E)$ are the same as those of $[\mathbf{F l}]$. By abuse of notation, we simply write $s_{n}(E)$ for $\operatorname{deg} s_{n}(E)$ when $n=\operatorname{dim} X$. We denote also by $H(E)$ the tautological line bundle $\mathcal{O}_{\mathbf{P}(E)}(1)$ on $\mathbf{P}(E)$. For a scheme $X$ over a scheme $Y$ and a line bundle $L$ on $X$, we denote by $P_{X / Y}^{1}(L)$ the bundle of principal parts of $L$ of first order on $X$ over $Y$. For a linear system $\Lambda, \mathrm{Bs} \Lambda$ denotes the base locus of $\Lambda$. Let $[r]$ denote the greatest integer not greater than $r$ for a real number $r$.

\section{Preliminaries and Proof of Proposition 0.1.}

Let $X$ be an $n$-dimensional nondegenerate closed submanifold in $\mathbf{P}^{N}$. Let $B$ be the blowing-up of $X \times X$ along the diagonal $\Delta$, and let $S_{0}=\{(x, y, u) \in$ $(X \times X \backslash \Delta) \times \mathbf{P}^{N} \mid x, y$, and $u$ are collinear $\}$. Since $\mathbf{P}\left(\Omega_{X}\right)$ is the exceptional divisor of $B$, we can identify $X \times X \backslash \Delta$ with $B \backslash \mathbf{P}\left(\Omega_{X}\right)$. Thus $S_{0}$ can be identified with a closed submanifold of $\left(B \backslash \mathbf{P}\left(\Omega_{X}\right)\right) \times \mathbf{P}^{N}$. We define $S$ to be the closure of $S_{0}$ in $B \times \mathbf{P}^{N}$. We call $S$ the complete secant bundle of $X$. Let $p: S \hookrightarrow B \times \mathbf{P}^{N} \rightarrow B$ be the first projection and $\sigma: S \hookrightarrow B \times$ $\mathbf{P}^{N} \rightarrow \mathbf{P}^{N}$ the second projection. Then $\operatorname{Sec} X=\operatorname{Im}(\sigma)$. For a point $u \in$ 
Sec $X$, let $\Sigma_{u}=\sigma\left(p^{-1}\left(p\left(\sigma^{-1}(u)\right)\right)\right), Q_{u}=\Sigma_{u} \cap X$, and $\theta_{u}=\{x \in X \mid u \in$ $\left.T_{x} X\right\}$. We call $\Sigma_{u}$ the secant cone, $Q_{u}$ the secant locus, and $\theta_{u}$ the tangent locus, with respect to $u$. Let $\operatorname{Sm}(\operatorname{Sec} X)$ denote the smooth locus of $\operatorname{Sec} X$. Let $\gamma: \operatorname{Sm}(\operatorname{Sec} X) \rightarrow G\left(\operatorname{dim} \operatorname{Sec} X, \mathbf{P}^{N}\right)$ be the Gauss map $u \mapsto T_{u} \operatorname{Sec} X$ of $\operatorname{Sm}(\operatorname{Sec} X)$. For a point $u \in \operatorname{Sm}(\operatorname{Sec} X), C_{u}$ denotes the closure of $\gamma^{-1}(\gamma(u))$ in $\operatorname{Sec} X$. Thus

$$
C_{u}=\overline{\left\{v \in \operatorname{Sm}(\operatorname{Sec} X) \mid T_{v} \operatorname{Sec} X=T_{u} \operatorname{Sec} X\right\}}
$$

and $C_{u}$ is called the contact locus of $\operatorname{Sec} X$ with $T_{u} \operatorname{Sec} X$. We fix and will use these notations in the following four sections.

We first observe that $\Sigma_{u}=u * Q_{u}$ and that $C_{u}$ is a linear subspace in $\mathbf{P}^{N}$ for a general point $u \in \operatorname{Sec} X$ (see, for example, [Z, Chap. 1, Theorem $2.3 \mathrm{c})]$ ). We also have $\Sigma_{u} \subseteq C_{u}$ for any general point $u \in$ $\operatorname{Sm}(\operatorname{Sec} X)$ (see, for example, $[\mathbf{F},(3.6)]$ or $\left[\mathbf{O h}\right.$, Corollary 1.2]). Let $D_{u}^{\prime}=$ $\overline{\cup_{v \in C_{u}} \text { : general } Q_{v}}$. Then $C_{u}=\operatorname{Sec} D_{u}^{\prime}$ and $1+2 \operatorname{dim} D_{u}^{\prime}=\operatorname{dim} Q_{u}+\operatorname{dim} C_{u}$ for any general point $u \in \operatorname{Sec} X$ (see [Oh, Lemma 1.4]). Let $X_{u}=\{x \in$ $\left.X \mid T_{x} X \subseteq T_{u} \operatorname{Sec} X\right\}$ for a point $u \in \operatorname{Sm}(\operatorname{Sec} X)$. Then $D_{u}^{\prime} \subseteq X_{u}$ for any general point $u \in \operatorname{Sec} X$ by [Oh, Corollary 1.3].

Lemma 1.1. For a general point $u \in \operatorname{Sec} X$, we have

1) $\operatorname{dim} Q_{u}=2 n+1-\operatorname{dim} \operatorname{Sec} X$

2) $\operatorname{dim} \Sigma_{u}=2 n+2-\operatorname{dim} \operatorname{Sec} X$;

3) $\operatorname{dim} X_{u} \leq \operatorname{dim} \operatorname{Sec} X-n-1$ if $\operatorname{Sec} X \neq \mathbf{P}^{N}$.

Proof. Note that $\sigma^{-1}(u) \rightarrow p_{i} \circ p\left(\sigma^{-1}(u)\right)$ is finite for a point $u \in \operatorname{Sec} X \backslash X$ where $p_{i}: B \rightarrow X$ is the $i$-th projection $(i=1,2)$. Thus we have $\operatorname{dim} Q_{u}=$ $\operatorname{dim} \sigma^{-1}(u)$ for a point $u \in \operatorname{Sec} X \backslash X$ since $Q_{u}=p_{i} \circ p\left(\sigma^{-1}(u)\right)$. Hence we have 1). Since $\operatorname{dim} \Sigma_{u}=\operatorname{dim} Q_{u}+1$, we have 2). Suppose that $\operatorname{Sec} X \neq \mathbf{P}^{N}$. Then $T_{u} \operatorname{Sec} X \neq \mathbf{P}^{N}$. Let $T\left(X_{u}, X\right)=\cup_{x \in X_{u}} T_{x} X$. By the definition of $X_{u}$, we obtain $T\left(X_{u}, X\right) \subseteq T_{u} \operatorname{Sec} X$. Let $S\left(X_{u}, X\right)=X_{u} * X$. Then $X \subseteq S\left(X_{u}, X\right)$. Note also that $X$ is not contained in $T_{u} \operatorname{Sec} X$ since $X$ is nondegenerate in $\mathbf{P}^{N}$. Therefore we get $\operatorname{dim} S\left(X_{u}, X\right)=\operatorname{dim} X_{u}+n+1$ by $\left[\mathbf{Z}\right.$, Chap. 1, Theorem 1.4]. Hence we have 3) since $\operatorname{dim} S\left(X_{u}, X\right) \leq$ $\operatorname{dim} \operatorname{Sec} X$.

Here let us recall the definition of degeneration of a secant or tangent variety (see $[\mathbf{L}]$ ).

Definition 1.2. Sec $X$ is said to be degenerate if $\operatorname{dim} \operatorname{Sec} X \leq 2 n$ and $\operatorname{Sec} X \neq \mathbf{P}^{N}$. Likewise Tan $X$ is said to be degenerate if $\operatorname{dim} \operatorname{Tan} X \leq 2 n-1$ and $\operatorname{Tan} X \neq \mathbf{P}^{N}$.

Now we give a proof of Proposition 0.1.

Proof of Proposition 0.1. Let $u$ be a general point of $\operatorname{Sec} X$. Note first that showing that $\operatorname{dim} \operatorname{Im}(\gamma)=2(\operatorname{dim} \operatorname{Sec} X-n-1-c)$ is equivalent to 
showing that $\operatorname{dim} C_{u}=2 n+2-\operatorname{dim} \operatorname{Sec} X+2 c$. Since $\Sigma_{u} \subseteq C_{u}$, we have $2 n+2-\operatorname{dim} \operatorname{Sec} X=\operatorname{dim} \Sigma_{u} \leq \operatorname{dim} C_{u}$ by Lemma 1.12$)$. On the other hand, $\operatorname{dim} C_{u}=1+2 \operatorname{dim} D_{u}^{\prime}-\operatorname{dim} Q_{u}=2 \operatorname{dim} D_{u}^{\prime}+(2-n+\varepsilon) / 2$ by Lemma 1.11$)$ and $2 \operatorname{dim} D_{u}^{\prime}+(2-n+\varepsilon) / 2 \leq 2 \operatorname{dim} X_{u}+(2-n+\varepsilon) / 2 \leq$ $2(\operatorname{dim} \operatorname{Sec} X-n-1)+((2-n+\varepsilon) / 2)$ by Lemma 1.13$)$. Thus $\operatorname{dim} C_{u} \leq$ $2 n+2-\operatorname{dim} \operatorname{Sec} X+2 \varepsilon$. Hence we have $\operatorname{dim} C_{u}=2 n+2-\operatorname{dim} \operatorname{Sec} X+2 c$ for some integer $c(0 \leq c \leq \varepsilon)$ because $\operatorname{dim} C_{u}$ depends only on $2 \operatorname{dim} D_{u}^{\prime}$. Therefore we have proved Proposition 0.1.

Remark 1.3. To the best of my knowledge, all examples of linearly normal manifolds $X \subset \mathbf{P}^{N}$ with degenerate secant varieties satisfy a condition that $\operatorname{dim} \operatorname{Im}(\gamma) \geq n$ (see Examples below). It is an unsolved problem whether there exist manifolds of $\operatorname{dim} \operatorname{Im}(\gamma)<n$ or not.

In order to see examples of projective manifolds with degenerate secant varieties, we state some propositions. First the following two propositions are fundamental.

Proposition 1.4. Let $H \subset \mathbf{P}^{N}$ be a hyperplane such that $X \cap H$ is nonsingular. If $\operatorname{dim} \operatorname{Sec} X \leq 2 n$, then $(\operatorname{Sec} X) \cap H=\operatorname{Sec}(X \cap H)$.

Proof. The inclusion $(\operatorname{Sec} X) \cap H \supseteq \operatorname{Sec}(X \cap H)$ is obvious. Let $u \in$ $((\operatorname{Sec} X) \cap H) \backslash(X \cap H)$. Since $\operatorname{dim} \operatorname{Sec} X \leq 2 n, Q_{u}$ is positive dimensional. Therefore $Q_{u} \cap H \neq \emptyset$. Let $x \in Q_{u} \cap H$. Then $x$ lies on either a secant line $x * y(y \in X, y \neq x)$ of $X$ or a tangent line $l_{x}$ of $X$ at $x$ either of which passes through $u$, since $X$ is regular. If $u \in x * y$, then $x \in X \cap H$ and $y \in Q_{u} \cap(u * x) \subseteq X \cap H$. Therefore $u \in \operatorname{Sec}(X \cap H)$. If $u \in l_{x}$, then $l_{x}=u * x \subseteq\left(T_{x} X\right) \cap H=T_{x}(X \cap H)$ since $X \cap H$ is nonsingular. Thus $l_{x}$ is a tangent line of $X \cap H$ at $x$. Hence $u \in \operatorname{Tan}(X \cap H) \subseteq \operatorname{Sec}(X \cap H)$.

Proposition 1.5. Let $Y \subseteq \mathbf{P}^{N}$ be a projective variety (not necessarily smooth) of dimension $n$ and let $H \subset \mathbf{P}^{N}$ be a general hyperplane. If $\operatorname{dim} \operatorname{Sec} Y=2 n+1$, then $\operatorname{dim} \operatorname{Sec}(Y \cap H)=2 n-1$.

Proof. Let $H \subset \mathbf{P}^{N}$ be a general hyperplane, and let $x, y \in Y \cap H$ be a general point. Then neither $T_{x} Y$ nor $T_{y} Y$ is contained in $H$. Since $\operatorname{dim} \operatorname{Sec} Y=2 n+1$, we have $T_{x} Y \cap T_{y} Y=\emptyset$ by Terracini's lemma (see, for example, [F-R, Lemma 2.1]). Therefore $T_{x}(Y \cap H) \cap T_{y}(Y \cap H)=\emptyset$, or equivalently $\operatorname{dim} T_{x}(Y \cap H) * T_{y}(Y \cap H)=2 n-1$. Hence again by Terracini's lemma we obtain $\operatorname{dim} \operatorname{Sec}(Y \cap H)=2 n-1$.

Terracini's lemma also implies the following proposition.

Proposition 1.6. Let $Y \subseteq \mathbf{P}^{N}$ be a projective variety (not necessarily smooth) of dimension $n$ and let $H \subset \mathbf{P}^{N}$ be a general hypersurface of degree $d \geq 2$.

1) If $\operatorname{dim} \operatorname{Sec} Y \leq 2 n-1$, then $\operatorname{Sec}(Y \cap H)=\operatorname{Sec} Y$.

2) If $\operatorname{dim} \operatorname{Sec} Y \geq 2 n$, then $\operatorname{dim} \operatorname{Sec}(Y \cap H)=2 n-1$. 
Proof. Let $X$ be the smooth locus of $Y$ and let $\delta=2 n+1-\operatorname{dim} \operatorname{Sec} Y$. Then by Terracini's lemma we have $\delta=1+\operatorname{dim} T_{p} X \cap T_{q} X$ for general two points $p, q$ of $X$, where we set $\operatorname{dim} \emptyset=-1$. If $T_{p} X \cap T_{q} X=\emptyset$ for general two points $p, q$ of $X$, then the statement obviously holds. Thus we assume in the following that $T_{p} X \cap T_{q} X \neq \emptyset$ for general two points $p, q$ of $X$. Let $U$ be the open subset of $X \times X \backslash \Delta_{X}$ which consists of points $(p, q)$ such that $\delta=1+\operatorname{dim} T_{p} X \cap T_{q} X$. Let

$$
\begin{aligned}
A & =\left\{(H, p, q) \in\left|\mathcal{O}_{\mathbf{P}^{N}}(d)\right| \times U \mid p, q \in H\right\}, \\
B_{1} & =\left\{(H, p, q) \in A \mid T_{p} X \cap T_{q} X \subseteq T_{p} H\right\}, \\
B_{2} & =\left\{(H, p, q) \in A \mid T_{p} X \cap T_{q} X \subseteq T_{q} H\right\}
\end{aligned}
$$

and

$$
C=\left\{(H, p, q) \in A \backslash\left(B_{1} \cup B_{2}\right) \mid T_{p} X \cap T_{q} X \cap T_{p} H=T_{p} X \cap T_{q} X \cap T_{q} H\right\} .
$$

Then $A$ is irreducible, $B_{1}$ and $B_{2}$ are closed in $A$ and $C$ is closed in $A \backslash$ $\left(B_{1} \cup B_{2}\right)$.

Since $A$ is irreducible, $B_{1} \cup B_{2}$ is a proper subset of $A$ if $B_{1}$ and $B_{2}$ are proper subsets of $A$. We claim that $B_{1}$ and $B_{2}$ are proper subsets of $A$. Let $(p, q) \in U$. The image of the rational map $H \mapsto T_{p} H$ from $\left|\mathcal{O}_{\mathbf{P}^{N}}(d)-p-q\right|$ to the dual projective space $\check{\mathbf{P}}^{N}$ is the hyperplane $\check{p}$ corresponding to the point $p$ since $d \geq 2$. Note that $\{p\} \neq T_{p} X \cap T_{q} X$ for a general point $(p, q) \in U$. Indeed, it is obvious if $X$ is linear since $n \geq 1$; thus assume that $X$ is not linear. Then the subset of $U$ consisting of points $(p, q)$ such that $\{p\}=$ $T_{p} X \cap T_{q} X$ is contained in the subset which consists of points $(p, q) \in U$ such that $p \in T_{q} X$, and this subset is a proper closed subset of $U$ since $X$ is not linear. Note also that the linear subset $\left(T_{p} X \cap T_{q} X\right)^{\vee}$ in $\check{\mathbf{P}}^{N}$ which is dual to $T_{p} X \cap T_{q} X$ is a proper subset of $\check{\mathbf{P}}^{N}$ because $T_{p} X \cap T_{q} X \neq \emptyset$. Thus $\check{p}$ is not contained in $\left(T_{p} X \cap T_{q} X\right)^{\vee}$. Hence, for a general point $(p, q) \in U$ and a general menber $H \in\left|\mathcal{O}_{\mathbf{P}^{N}}(d)-p-q\right|, T_{p} H$ does not contain $T_{p} X \cap T_{q} X$. Therefore $B_{1}$ is a proper subset of $A$. Similar argument shows that $B_{2}$ is a proper subset of $A$.

Suppose that $\delta=1$. Then we have $T_{p} X \cap T_{q} X \cap T_{p} H \cap T_{q} H=\emptyset$ for a point $(H, p, q) \in A \backslash\left(B_{1} \cup B_{2}\right)$. Moreover for a general point $(H, p, q) \in A \backslash\left(B_{1} \cup B_{2}\right)$, $X \cap H$ is nonsingular, $T_{p}(X \cap H)=T_{p} X \cap T_{p} H$ and $T_{q}(X \cap H)=T_{q} X \cap T_{q} H$. Hence $\operatorname{dim} \operatorname{Sec}(X \cap H)=2 n-1$ and this completes the proof of 2) of the proposition.

Suppose that $\delta \geq 2$. Thus we have $\operatorname{dim} T_{p} X \cap T_{q} X \geq 1$ for a point $(p, q) \in U$.

We claim that $C$ is a proper subset of $A \backslash\left(B_{1} \cup B_{2}\right)$. Let $f$ be the rational map $H \mapsto\left(T_{p} H, T_{q} H\right)$ from $(p, q) \times\left|\mathcal{O}_{\mathbf{P}^{N}}(d)-p-q\right|$ to $\check{\mathbf{P}}^{N} \times \check{\mathbf{P}}^{N}$. The image of $f$ is $\check{p} \times \check{q}$ since $d \geq 2$. Let $(\check{p} \times \check{q})^{\circ}$ be the set consisting of points $(F, G) \in \check{p} \times \check{q}$ such that neither $F$ nor $G$ contains $T_{p} X \cap T_{q} X$. Then the image of $A \backslash\left(B_{1} \cup B_{2}\right) \cap(p, q) \times\left|\mathcal{O}_{\mathbf{P}^{N}}(d)-p-q\right|$ is contained in $(\check{p} \times \check{q})^{\circ}$. 
Note that the closed subset in $(\check{p} \times \check{q})^{\circ}$ consisting of points $(F, G)$ such that $T_{p} X \cap T_{q} X \cap F=T_{p} X \cap T_{q} X \cap G$ is a proper subset of $(\check{p} \times \check{q})^{\circ}$ because $\operatorname{dim} T_{p} X \cap T_{q} X \geq 1$. Therefore $C$ is a proper subset of $A \backslash\left(B_{1} \cup B_{2}\right)$.

Hence we have $\operatorname{dim} T_{p} X \cap T_{q} X \cap T_{p} H \cap T_{q} H=\operatorname{dim} T_{p} X \cap T_{q} X-2$ and $X \cap H$ is nonsingular for a general point $(H, p, q) \in A \backslash\left(B_{1} \cup B_{2} \cup C\right)$. Therefore $\operatorname{dim} \operatorname{Sec}(X \cap H)=\operatorname{dim} \operatorname{Sec} X$.

The following proposition is useful when one computes the dimension of the image of the Gauss map of the secant variety of a hyperplane section of some known manifold.

Proposition 1.7. Let $Y \subset \mathbf{P}^{N}$ be a projective variety of dimension $m$. For a regular point $u$ of $Y$, let $C_{u, Y}$ denote the contact locus of $Y$ with $T_{u} Y$. Then for a general hyperplane $H \subset \mathbf{P}^{N}$ and a general point $u \in Y \cap H$, $C_{u, Y} \cap H=C_{u, Y \cap H}$.

Proof. The inclusion $C_{u, Y} \cap H \subseteq C_{u, Y \cap H}$ is easy.

In order to prove the other inclusion, we claim the following. Let $H$ be a general hyperplane of $\mathbf{P}^{N}$ and $u$ a general regular point of $Y \cap H$. If $v$ is a regular point of $Y \cap H$ such that $\operatorname{dim} T_{u} Y \cap T_{v} Y=m-1$ and $u$, $v \in T_{u} Y \cap T_{v} Y$, then $T_{u} Y \cap T_{v} Y$ is not contained in $H$.

The other inclusion is straightforward from this claim. Indeed let $v$ be a regular point of $Y \cap H$ such that $T_{u}(Y \cap H)=T_{v}(Y \cap H)$. Then $v$ is a regular point of $Y$ and $\left(T_{u} Y\right) \cap H=\left(T_{v} Y\right) \cap H$. Hence $\left(T_{u} Y\right) \cap H=\left(T_{u} Y\right) \cap$ $\left(T_{v} Y\right) \cap H \subseteq T_{u} Y \cap T_{v} Y$. If $T_{u} Y \neq T_{v} Y$, then $T_{u} Y \cap T_{v} Y=T_{u} Y \cap H \subset H$ and this contradicts the claim. Therefore $T_{u} Y=T_{v} Y$.

Now we prove the claim. We may assume that $Y$ is not linear because, otherwise, the proposition is obvious. Let

$\mathcal{Y}=\left\{(u, v) \in Y_{\text {reg }} \times Y_{\text {reg }} \mid \operatorname{dim} T_{u} Y \cap T_{v} Y=m-1\right.$ and $\left.u, v \in T_{u} Y \cap T_{v} Y\right\}$.

Then $\operatorname{dim} \mathcal{Y} \leq 2 m-1$. Let

$$
\mathcal{Z}=\left\{((u, v), H) \in \mathcal{Y} \times \check{\mathbf{P}}^{N} \mid T_{u} Y \cap T_{v} Y \subseteq H\right.
$$

and neither $T_{u} Y$ nor $T_{v} Y$ is contained in $\left.H\right\}$.

Since $\operatorname{dim} T_{u} Y \cap T_{v} Y=m-1$, we get $\operatorname{dim} \mathcal{Z}=\operatorname{dim} \mathcal{Y}+N-m \leq m+N-1$. Let

$$
\mathcal{W}=\left\{(u, H) \in Y_{\text {reg }} \times \check{\mathbf{P}}^{N} \mid u \in H \text { and } T_{u} Y \text { is not contained in } H\right\} .
$$

Then $\operatorname{dim} \mathcal{W}=m+N-1$. Let $f: \mathcal{Z} \rightarrow \mathcal{W}$ be the natural projection. Our claim is to say that $f$ is not dominant. Suppose to the contrary that $f$ is dominant. Then $f$ is quasi-finite. Note that a general fiber of $f$ is a dense subset of $C_{u, Y \cap H} \backslash\left(C_{u, Y} \cap H\right)$. Note also that $C_{u, Y \cap H}$ is irreducible since char. $k=0$. Hence $\operatorname{dim} C_{u, Y \cap H}=0$. On the other hand, $C_{u, Y} \cap H$ is not empty, so that $\operatorname{dim} C_{u, Y \cap H} \geq 1$. This is a contradiction. This completes the proof of the claim. 
Here we give examples of projective manifolds with degenerate secant varieties, and see the value of the invariant $c$ in Proposition 0.1 for each example.

Example 1. Let $X=\mathbf{P}^{l} \times \mathbf{P}^{n-l}$ embedded by the Segre embedding in $\mathbf{P}^{(l+1)(n-l+1)-1}$ where $2 \leq l \leq[n / 2]$. Then $\operatorname{dim} \operatorname{Sec} X=2 n-1$ and $\operatorname{Sec} X$ is degenerate. In this case $\operatorname{dim} C_{u}=2 n+2-\operatorname{dim} \operatorname{Sec} X$ for any general point $u \in \operatorname{Sec} X$. These facts are shown by Terracini's lemma ([F-R, Lemma 2.1]). If $l=[n / 2]$, then $X$ is a Scorza variety.

Example 2. Let $X=\mathbf{P}_{\mathbf{P}^{l}}(\mathcal{E})$ embedded in $\mathbf{P}\left(H^{0}(\mathcal{E})\right)$ by the tautological line bundle $H(\mathcal{E})$ of $X$ where $\mathcal{E}=\mathcal{O}(1)^{\oplus(n+1-2 l)} \oplus T_{\mathbf{P}^{l}}$ and $2 \leq l \leq$ $[(n+1) / 2]$. Then $\operatorname{dim} \operatorname{Sec} X=2 n$ and $\operatorname{Sec} X$ is degenerate. In this case $\operatorname{dim} C_{u}=2 n+2-\operatorname{dim} \operatorname{Sec} X$ for any general point $u \in \operatorname{Sec} X$. One can see these facts by Propositions 1.4 and 1.7 because $X$ is a smooth hyperplane section of the Segre manifold in Example 1.

Example 3. Let $X=\mathbf{P}_{\mathbf{P}^{l}}(\mathcal{E})$ embedded in $\mathbf{P}\left(H^{0}(\mathcal{E})\right)$ by the tautological line bundle $H(\mathcal{E})$ of $X$ where $\mathcal{E}=\mathcal{O}(1)^{\oplus(n-l)} \oplus \mathcal{O}(2)$ and $2 \leq l \leq n$. Then $\operatorname{dim} \operatorname{Sec} X=2 n$ and $\operatorname{Sec} X$ is degenerate. In this case $\operatorname{dim} C_{u}=$ $2 n+2-\operatorname{dim} \operatorname{Sec} X$ for any general point $u \in \operatorname{Sec} X$. These facts are shown by Terracini's lemma. If $l=n$, then $X$ is a Scorza variety.

Example 4. Let $G$ be a simple algebraic group of rank $\geq 2$ and $\boldsymbol{g}$ its Lie algebra. Let $X$ be the closed orbit of the action on $\mathbf{P}_{*}(\boldsymbol{g})$ by $G$ action induced by the adjoint representation $G \rightarrow G L(\boldsymbol{g})$. We call $X$ the adjoint manifold of $G$ in $\mathbf{P}_{*}(\boldsymbol{g})$. Then Sec $X$ is degenerate and $2 n$-dimensional, and $\operatorname{dim} C_{u}=2 n+2-\operatorname{dim} \operatorname{Sec} X$ for any general point $u \in \operatorname{Sec} X$ by [K-O-Y].

Example 5. Let $X=G\left(1, \mathbf{P}^{(n+2) / 2}\right)$ in $\mathbf{P}^{N}$ via the Plücker embedding where $n$ is an even integer $\geq 8$. Then $\operatorname{dim} \operatorname{Sec} X=2 n-3$ and $\operatorname{Sec} X$ is degenerate. In this case $\operatorname{dim} C_{u}=2 n+2-\operatorname{dim} \operatorname{Sec} X$ for any general point $u \in \operatorname{Sec} X$. As above, Terracini's lemma shows these facts. In this case, $X$ is a Scorza variety.

Example 6. Let $X \subset \mathbf{P}^{26}$ be the 16-dimensional Severi variety (see $[\mathbf{Z}$, Chap. 3, 2.5.E)] or $[\mathbf{L}-\mathbf{V}, \S 1 \mathrm{~b}])$. Then $\operatorname{dim} \operatorname{Sec} X=25=2 n-7$ and $\operatorname{Sec} X$ is degenerate. In this case $\operatorname{dim} C_{u}=2 n+2-\operatorname{dim} \operatorname{Sec} X$ for any general point $u \in \operatorname{Sec} X$ by Proposition 0.1 since $X$ is a Severi variety.

In the following examples, let $c(X)$ denote the invariant $c$ in Proposition 0.1 for a projective manifold $X \subset \mathbf{P}^{N}$, and let $\delta(X)$ denote the secant defect $2 n+1-\operatorname{dim} \operatorname{Sec} X$ of $X$. 
Example 7. Let $Y \subset \mathbf{P}^{N+1}$ be an $(n+1)$-dimensional projective manifold with degenerate secant variety of $\operatorname{dim} \operatorname{Sec} Y \leq 2(n+1)-1$. Let $X \subset \mathbf{P}^{N}$ be a general smooth hyperplane section of $Y \subset \mathbf{P}^{N+1}$. Then $\operatorname{dim} \operatorname{Sec} X=\operatorname{dim} \operatorname{Sec} Y-1 \leq 2 n$ (i.e., $\delta(X)=\delta(Y)-1$ ) by Proposition 1.4 and Sec $X$ is also degenerate. We have also $c(X)=c(Y)$ by Proposition 1.7.

Example 8. Let $Y \subset \mathbf{P}^{N}$ be an $(n+1)$-dimensional projective manifold with degenerate secant variety of $\operatorname{dim} \operatorname{Sec} Y \leq 2(n+1)-2$. Let $H \subset \mathbf{P}^{N}$ be a general hypersurface of degree $d \geq 2$, and let $X=Y \cap H$. Then $X \subset \mathbf{P}^{N}$ is an $n$-dimensional nondegenerate projective manifold with degenerate secant variety $\operatorname{Sec} X$ equal to $\operatorname{Sec} Y$ by Proposition 1.61 ). Hence $\delta(X)=\delta(Y)-2$ and $c(X)=c(Y)+1>0$.

Example 9. Let $Y \subset \mathbf{P}^{N-1}$ be an $n$-dimensional projective manifold with degenerate secant variety of $\operatorname{dim} \operatorname{Sec} Y \leq 2 n-1$. Embed $\mathbf{P}^{N-1}$ in $\mathbf{P}^{N}$ as a hyperplane and let $p \in \mathbf{P}^{N} \backslash \mathbf{P}^{N-1}$. Let $C_{p}(Y)$ denote the cone over $Y$ with vertex $p$. Let $X$ be a smooth intersection of $C_{p}(Y)$ and a general hypersurface of $\mathbf{P}^{N}$ of degree $d \geq 2$. Then $X \subset \mathbf{P}^{N}$ is an $n$-dimensional nondegenerate projective manifold with degenerate secant variety $\operatorname{Sec} X$ equal to $C_{p}(\operatorname{Sec} Y)$ by Proposition 1.61 ). Hence $\delta(X)=\delta(Y)-1$ and $c(X)=c(Y)+1>0$.

\section{Proof of Theorem 0.2 .}

In this section, we investigate $n$-dimensional projective manifolds with degenerate secant varieties under an additional condition that $\operatorname{dim} \operatorname{Im}(\gamma)=$ $2(\operatorname{dim} \operatorname{Sec} X-n-1)$, and prove Theorem 0.2.

Let $X$ be an $n$-dimensional nondegenerate projective manifold in $\mathbf{P}^{N}$ with degenerate secant variety $\operatorname{Sec} X$ in this section, unless otherwise stated. Note that the condition that $\operatorname{dim} \operatorname{Im}(\gamma)=2(\operatorname{dim} \operatorname{Sec} X-n-1)$ is equivalent to the condition that $\operatorname{dim} C_{u}=2 n+2-\operatorname{dim} \operatorname{Sec} X$ for a general point $u \in \operatorname{Sec} X$. We begin with the following geometric observation.

Proposition 2.1. Assume that $\operatorname{dim} C_{u}=2 n+2-\operatorname{dim} \operatorname{Sec} X$ for a general point $u \in \operatorname{Sec} X$. Then the secant cone $\Sigma_{u}$ is a linear subspace of $\mathbf{P}^{N}$ of dimension $2 n+2-\operatorname{dim} \operatorname{Sec} X$ for any general point $u \in \operatorname{Sec} X$. Moreover the secant locus $Q_{u}$ is a smooth hyperquadric in $\Sigma_{u}$, and the tangent locus $\theta_{u}$ is a smooth hyperplane section of $Q_{u}$ for any general point $u \in \operatorname{Sec} X$.

Proof. The first statement follows immediately from Lemma 1.12 ) and the fact that $\Sigma_{u} \subseteq C_{u}$. For a proof of the second statement, note first that a linear subspace $\Sigma_{u}$ contains $Q_{u}$ as a hypersurface. Second note that $X$ is not a hypersurface in $\mathbf{P}^{N}$ because $\operatorname{Sec} X$ is degenerate, so that the trisecant lemma $[\mathbf{F},(1.6)]$ shows that $Q_{u}$ is a hyperquadric in $\Sigma_{u}$. For the rest of the 
second statement, refer to the proof of Theorem 3 in $[\mathbf{F}-\mathbf{R}$, p. $964,1.15-$ p. 967, 1.7], and make obvious adjustments.

Corollary 2.2. If $\operatorname{dim} \operatorname{Sec} X \leq 2 n$ and $\operatorname{dim} \operatorname{Im}(\gamma)=2(\operatorname{dim} \operatorname{Sec} X-n-1)$, then $X$ is rationally connected. In particular $\kappa(X)=-\infty$ and $h^{i}\left(\mathcal{O}_{X}\right)=0$ for all $i>0$.

Proof. For the definition of rational connectedness, see [Ko-Mi-Mo, (2.2)]. If $\operatorname{dim} \operatorname{Sec} X \leq 2 n$, then $\operatorname{dim} Q_{u} \geq 1$ for a general point $u \in \operatorname{Sec} X$ by Lemma 1.11 ). Thus general two points can be joined by $Q_{u}$ which is quadric by the proposition above. Hence $X$ is rationally connected. The rest of the assertion follows immediately from [M-M, Theorem 1] and [Ko-Mi-Mo, (2.5.2)].

Remark 2.3. So far as I know, all examples of projective manifolds with degenerate secant varieties which satisfy the condition $\operatorname{dim} \operatorname{Im}(\gamma)=$ $2(\operatorname{dim} \operatorname{Sec} X-n-1)$ are not only rationally connected but also Fano. It is an unsolved problem whether such manifolds are always Fano or not.

The following proposition is a generalization of [F, Lemma (2.3)], where $n=3$, to arbitrary dimension $n$.

Proposition 2.4. Assume that $\operatorname{dim} C_{u}=2 n+2-\operatorname{dim} \operatorname{Sec} X$ for a general point $u \in \operatorname{Sec} X$. If $\operatorname{dim} \operatorname{Sec} X=2 n$, then $K_{X} \cdot Q_{u}=-n-1$ for a general point $u \in \operatorname{Sec} X$.

Proof. We will use the notation introduced in $\S 1$ before Lemma 1.1. First of all, let us recall another construction of the complete secant bundle $S$ of $X$ (see $[\mathbf{F},(1.2)]$ ). Let $p_{i}: B \rightarrow X$ be the $i$-th projection $(i=1,2)$. Let $L$ denote $\mathcal{O}_{X}(1)$, and let $\varphi: \mathcal{O}_{X} \otimes H^{0}(L) \rightarrow L$ be the natural surjection. Put $V=p_{1}^{*} L \oplus p_{2}^{*} L$ and let $\Phi=\left(p_{1}^{*} \varphi, p_{2}^{*} \varphi\right): \mathcal{O}_{B} \otimes H^{0}(L) \rightarrow V$ the morphism induced from $p_{1}^{*} \varphi$ and $p_{2}^{*} \varphi$. Let $W=\mathbf{P}(V)$, and let $f: W \rightarrow B$ be the projection. Then $f^{-1}\left(\mathbf{P}\left(\Omega_{X}\right)\right)=\mathbf{P}^{1} \times \mathbf{P}\left(\Omega_{X}\right)$. We define homogeneous coordinates $\left(e_{1} ; e_{2}\right)$ of $\mathbf{P}^{1}$ of $\mathbf{P}^{1} \times \mathbf{P}\left(\Omega_{X}\right)$ by the formula $V=p_{1}^{*} L e_{1} \oplus p_{2}^{*} L e_{2}$. Let $\mathcal{O}_{W}(1)$ denote $\mathcal{O}_{\mathbf{P}(V)}(1)$. The composite of $f^{*} \Phi: \mathcal{O}_{W} \otimes H^{0}(L) \rightarrow f^{*} V$ and $f^{*} V \rightarrow \mathcal{O}_{W}(1)$ defines a linear system $\Lambda$ on $W$. We have $\operatorname{Bs} \Lambda=\{(1 ;-1)\} \times$ $\mathbf{P}\left(\Omega_{X}\right) \subset f^{-1}\left(\mathbf{P}\left(\Omega_{X}\right)\right)$. Let $g: W^{\prime} \rightarrow W$ be the blowing-up along Bs $\Lambda$. Let $E$ be the exceptional divisor of $g$, and $D$ the proper transform of $\mathbf{P}^{1} \times \mathbf{P}\left(\Omega_{X}\right)$ along $g$. Then $D$ is contractible, and we obtain a morphism $\tau: W^{\prime} \rightarrow S$ such that $\tau$ is the blowing-up of $S$ along $\tau(D)$ and $D=\tau^{-1}(\tau(D))$. We also have $\tau^{*} \sigma^{*} \mathcal{O}_{\mathbf{P}^{N}}(1)=g^{*} \mathcal{O}_{W}(1) \otimes \mathcal{O}(-E)$.

Let $u \in \operatorname{Sec} X$ be a general point. Since $Q_{u}$ is a smooth quadric by Proposition 2.1 , we have $\sigma^{-1}(u) \cong Q_{u}$ via $p_{i} \circ p$. Thus we also have $\sigma^{-1}(u) \cap$ $p^{-1}\left(\mathbf{P}\left(\Omega_{X}\right)\right) \cong \theta_{u}$ via $p_{i} \circ p$, and hence we have $\left.p^{*} \mathcal{O}_{B}\left(\mathbf{P}\left(\Omega_{X}\right)\right)\right|_{\sigma^{-1}(u)} \cong$ $\mathcal{O}_{Q_{u}}(1)$ by Proposition 2.1. Since $\tau(D)$ is a section of $p^{-1}\left(\mathbf{P}\left(\Omega_{X}\right)\right) \rightarrow \mathbf{P}\left(\Omega_{X}\right)$ and, for any point $\left[t_{x}\right] \in \mathbf{P}\left(\Omega_{X}\right), p^{-1}\left(\left[t_{x}\right]\right) \cap \tau(D)$ corresponds to the tangent point $x \in X$ in the tangent line $t_{x}$, we get $D \cap \tau^{-1}\left(\sigma^{-1}(u)\right)=\emptyset$ 
and $\tau^{-1}\left(\sigma^{-1}(u)\right) \cong \sigma^{-1}(u)$. Since $\mathcal{O}=\left.\sigma^{*} \mathcal{O}_{\mathbf{P}^{N}}(1)\right|_{\sigma^{-1}(u)} \cong g^{*} \mathcal{O}_{W}(1) \otimes$ $\left.\mathcal{O}(-E)\right|_{\tau^{-1}\left(\sigma^{-1}(u)\right)}$, we have $\left.\mathcal{O}(E)\right|_{\tau^{-1}\left(\sigma^{-1}(u)\right)}=\left.g^{*} \mathcal{O}_{W}(1)\right|_{\tau^{-1}\left(\sigma^{-1}(u)\right)}$. Thus

$$
\begin{aligned}
& \mathcal{O}_{Q_{u}}(1)\left.\cong p^{*} \mathcal{O}_{B}\left(\mathbf{P}\left(\Omega_{X}\right)\right)\right|_{\sigma^{-1}(u)} \\
&\left.\cong(E+D)\right|_{\tau^{-1}\left(\sigma^{-1}(u)\right)} \\
& \\
&=\left.E\right|_{\tau^{-1}\left(\sigma^{-1}(u)\right)} \\
&=\left.g^{*} \mathcal{O}_{W}(1)\right|_{\tau^{-1}\left(\sigma^{-1}(u)\right)}
\end{aligned}
$$

Note that $\left.K_{S}\right|_{\sigma^{-1}(u)}=K_{\sigma^{-1}(u)} \cong K_{Q_{u}}$ by generic smoothness. Therefore we have

$$
\begin{aligned}
\left.g^{*} K_{W}\right|_{\tau^{-1}\left(\sigma^{-1}(u)\right)} & =\left.\left(K_{W^{\prime}}-E\right)\right|_{\tau^{-1}\left(\sigma^{-1}(u)\right)} \\
& =\left.\left(\tau^{*} K_{S}+D-E\right)\right|_{\tau^{-1}\left(\sigma^{-1}(u)\right)} \\
& \cong \mathcal{O}\left(K_{Q_{u}}\right) \otimes \mathcal{O}_{Q_{u}}(-1) \\
& =\mathcal{O}_{Q_{u}}\left(-\operatorname{dim} Q_{u}-1\right)
\end{aligned}
$$

and

$$
\begin{aligned}
& \left.g^{*} K_{W}\right|_{\tau^{-1}\left(\sigma^{-1}(u)\right)} \\
& =g^{*}\left(\mathcal { O } _ { W } ( - 2 ) \otimes f ^ { * } \left(\mathcal{O}\left(p_{1}^{*} K_{X}+p_{2}^{*} K_{X}+(n-1) \mathbf{P}\left(\Omega_{X}\right)\right) \otimes p_{1}^{*} L \otimes\right.\right. \\
& \left.\left.\quad p_{2}^{*} L\right)\right)\left.\right|_{\tau^{-1}\left(\sigma^{-1}(u)\right)} \\
& \cong \\
& \left.\mathcal{O}_{Q_{u}}(-2) \otimes \mathcal{O}\left(K_{X}\right)\right|_{p_{1}\left(p\left(\sigma^{-1}(u)\right)\right)} \otimes \iota^{*}\left(\left.\mathcal{O}\left(K_{X}\right)\right|_{p_{2}\left(p\left(\sigma^{-1}(u)\right)\right)}\right) \otimes \\
& \quad \mathcal{O}_{Q_{u}}((n-1)+1+1) \\
& =\mathcal{O}\left(\left.K_{X}\right|_{Q_{u}}\right) \otimes \iota^{*} \mathcal{O}\left(\left.K_{X}\right|_{Q_{u}}\right) \otimes \mathcal{O}_{Q_{u}}(n-1)
\end{aligned}
$$

where $\iota: Q_{u}=p_{1}\left(p\left(\sigma^{-1}(u)\right)\right) \rightarrow p\left(\sigma^{-1}(u)\right) \rightarrow p_{2}\left(p\left(\sigma^{-1}(u)\right)\right)=Q_{u}$ is an automorphism of $Q_{u}$. Hence we get

$$
\mathcal{O}\left(\left.K_{X}\right|_{Q_{u}}+\iota^{*}\left(\left.K_{X}\right|_{Q_{u}}\right)\right) \cong \mathcal{O}_{Q_{u}}\left(-\operatorname{dim} Q_{u}-n\right) .
$$

If $\operatorname{dim} \operatorname{Sec} X=2 n$, then $\operatorname{dim} Q_{u}=1$ and $\mathcal{O}\left(\left.K_{X}\right|_{Q_{u}}\right) \cong \mathcal{O}\left(\iota^{*}\left(\left.K_{X}\right|_{Q_{u}}\right)\right)$, and we have $\operatorname{deg} \mathcal{O}_{Q_{u}}(1)=2$ by Proposition 2.1. Therefore $K_{X} \cdot Q_{u}=-n-1$.

We will recall some results of $\mathrm{H}$. Tango $[\mathbf{T}]$ in order to prove Theorem 0.2 $2)-4$ ) according to the idea of Fujita-Roberts-Tango.

Definition 2.5. For each integer $m$, we define a sequence

$$
1=f_{0}(m), f_{1}(m), f_{2}(m), \cdots, f_{r}(m), \cdots
$$

of rational numbers by the following equation in the formal power series ring $\mathbf{Q}[[t]]$ with coefficients in the field $\mathbf{Q}$ of rational numbers.

$$
\left(\sum_{i \geq 0} f_{i}(m) t^{i}\right)\left(\sum_{i \geq 0} f_{i}(m)(1-t)^{-i} t^{i}\right)=(1-t)^{-m} .
$$


We also define a sequence

$$
1=a_{0}(m), a_{1}(m), a_{2}(m), \cdots, a_{r}(m), \cdots
$$

in $\mathbf{Q}$ by the following equation in $\mathbf{Q}[[t]]$.

$$
\left(\sum_{i \geq 0} a_{i}(m) t^{i}\right)\left(\sum_{i \geq 0} a_{i}(m)(1-t)^{2 m-i} t^{i}\right)=(1-t)^{m}(1-2 t)^{-1} .
$$

Lemma 2.6. (1) If $l$ is an odd positive integer, then $f_{2^{m+1}-2}\left(2^{m} l\right)$ is not an integer for each $m \geq 1$.

(2) $a_{j}(m)=\sum_{i=0}^{j} f_{i}(m)$ for all $j \geq 0$.

(3) $\left(\sum_{i \geq 0}(-1)^{i} a_{i}(m) t^{i}\right)^{-1}=\sum_{i \geq 0}\left(f_{i}(m)+f_{i-1}(m)\right) t^{i}$ where $f_{-1}(m)=0$.

For a proof, see [T, Lemmas 2, 3, and 4].

Let $E=P_{X}^{1}\left(\mathcal{O}_{X}(1)\right)$, where $P_{X}^{1}\left(\mathcal{O}_{X}(1)\right)$ is the bundle of the principal parts of $\mathcal{O}_{X}(1)$ of first order, and let $\Theta=\mathbf{P}(E)$. Let us denote by $f$ : $\Theta \rightarrow X$ and by $g: \Theta \rightarrow \operatorname{Tan} X$ the morphisms induced from the projections $\Theta \hookrightarrow X \times \mathbf{P}^{N} \rightarrow X$ and $\Theta \hookrightarrow X \times \mathbf{P}^{N} \rightarrow \mathbf{P}^{N}$ respectively. Let $\beta_{u}: \theta_{u} \hookrightarrow X$ be the inclusion where $u$ is any point on $\operatorname{Tan} X$.

Lemma 2.7. Let $X$ be a closed submanifold in $\mathbf{P}^{N}$ over an algebraically closed field $k$ of characteristic 0 . Then

$$
c\left(\Omega_{\theta_{u}}^{1}\right)=c\left(\beta_{u}^{*} E \otimes \mathcal{O}_{\theta_{u}}(-1)\right) c\left(\beta_{u}^{*} E\right)
$$

for any general point $u \in \operatorname{Tan} X$.

For a proof, see [T, Lemma 6].

In the rest of this section, let $\varepsilon=2 \operatorname{dim} \operatorname{Sec} X-3 n-2$.

Proposition 2.8. Let $u$ be a general point in $\operatorname{Sec} X$. Suppose that $\operatorname{dim} C_{u}=$ $2 n+2-\operatorname{dim} \operatorname{Sec} X$. Then

(1) $c_{i}\left(\beta_{u}^{*} E\right)=a_{i}((n+\varepsilon) / 2) h_{u}^{i}$ for all $i(0 \leq i \leq(n-\varepsilon-2) / 2)$, where $h_{u}=c_{1}\left(\mathcal{O}_{\theta_{u}}(1)\right)$.

(2) $c_{i}\left(\beta_{u}^{*} N_{X / \mathbf{P}^{n}}(-1)\right)=\left(f_{i}((n+\varepsilon) / 2)+f_{i-1}((n+\varepsilon) / 2)\right) h_{u}^{i}$ for all $i(0 \leq i \leq$ $(n-\varepsilon-2) / 2)$.

(3) $f_{i}((n+\varepsilon) / 2)$ is an integer for all $i(0 \leq i \leq(n-\varepsilon-2) / 4)$.

(4) $f_{i}((n+\varepsilon) / 2)+f_{i-1}((n+\varepsilon) / 2) \geq 0$ for all $i(0 \leq i \leq(n-\varepsilon-2) / 2)$.

Proof. Put $F=\theta_{u}, h=h_{u}, \beta=\beta_{u}$, and $c_{i}=c_{i}\left(\beta_{u}^{*} E\right)$.

(1) Since $2 n+2-\operatorname{dim} \operatorname{Sec} X=(n-\varepsilon+2) / 2, F$ is a smooth quadric hypersurface of a linear space $\mathbf{P}^{(n-\varepsilon) / 2}$ by Proposition 2.1. Thus $c\left(\Omega_{F}\right)=$ $(1-h)^{(n-\varepsilon+2) / 2}(1-2 h)^{-1}$. Since $c\left(\beta^{*} E\right) c\left(\beta^{*} E \otimes \mathcal{O}_{F}(-1)\right)=c\left(\Omega_{F}\right)$ by 
Lemma 2.7, we have

$$
\left(\sum_{i \geq 0} c_{i}\right)\left(\sum_{i \geq 0} c_{i}(1-h)^{n+1-i}\right)=(1-h)^{(n-\varepsilon+2) / 2}(1-2 h)^{-1} .
$$

Put $m=(n+\varepsilon) / 2$. Then $n+\varepsilon=2 m$. Multiplying the formula above by $(1-h)^{\varepsilon-1}$, we get

$$
\left(\sum_{i \geq 0} c_{i}\right)\left(\sum_{i \geq 0} c_{i}(1-h)^{2 m-i}\right)=(1-h)^{m}(1-2 h)^{-1} .
$$

Thus, by virtue of the definition of $a_{i}(m)$, we have $c_{i}=a_{i}((n+\varepsilon) / 2) h^{i}$ for $0 \leq i \leq \operatorname{dim} F$.

(2) It follows from (1) that $c\left(\beta^{*} E^{\vee}\right)=\sum_{i>0}(-1)^{i} a_{i}((n+\varepsilon) / 2) h^{i}$. Recall that there is the following natural exact sequence of vector bundles.

$$
0 \rightarrow \beta^{*} E^{\vee} \rightarrow \mathcal{O}_{F} \otimes H^{0}\left(\mathcal{O}_{\mathbf{P}^{N}}(1)\right)^{\vee} \rightarrow \beta^{*} N_{X / \mathbf{P}^{N}}(-1) \rightarrow 0 .
$$

Applying Lemma 2.6 (3), we obtain

$$
\begin{aligned}
c\left(\beta^{*} N_{X / \mathbf{P}^{N}}(-1)\right) & =c\left(\beta^{*} E^{\vee}\right)^{-1} \\
& =\left(\sum_{i=0}^{\operatorname{dim} F}(-1)^{i} a_{i}((n+\varepsilon) / 2) h^{i}\right)^{-1} \\
& =\sum_{i=0}^{\operatorname{dim} F}\left(f_{i}((n+\varepsilon) / 2)+f_{i-1}((n+\varepsilon) / 2)\right) h^{i} .
\end{aligned}
$$

(3) Let $i$ be an integer such that $0 \leq 2 i \leq \operatorname{dim} F$. Then $0 \leq i \leq(n-\varepsilon-$ $2) / 4$. Since the intersection number $c_{i}^{2} h^{\operatorname{dim} \bar{F}-2 i}=2 a_{i}((n+\varepsilon) / 2)^{2}$ is an integer, $a_{i}((n+\varepsilon) / 2)$ must be an integer. Hence it follows from Lemma 2.6 (2) that $f_{i}((n+\varepsilon) / 2)$ is an integer for each $i(0 \leq i \leq(n-\varepsilon-2) / 4)$.

(4) Since $\beta^{*} N_{X / \mathbf{P}^{N}}(-1)$ is a spanned vector bundle, $c_{i}\left(\beta^{*} N_{X / \mathbf{P}^{N}}(-1)\right)$ is numerically non-negative for each $i(0 \leq i \leq \operatorname{dim} F)$. Therefore, by (2), $f_{i}((n+\varepsilon) / 2)+f_{i-1}((n+\varepsilon) / 2) \geq 0$ for each $i(0 \leq i \leq(n-\varepsilon-2) / 2)$.

Proposition 2.9. Assume that $\operatorname{dim} C_{u}=2 n+2-\operatorname{dim} \operatorname{Sec} X$ for a general point $u \in \operatorname{Sec} X$.

(1) If $\operatorname{dim} \operatorname{Sec} X=2 n-1$ (i.e., $\varepsilon=n-4$ ), then $K_{X} \cdot \theta_{u}=-n-2$.

(2) If $\operatorname{dim} \operatorname{Sec} X \leq 2 n-2$ (i.e., $\varepsilon \leq n-6)$, then $n+1 \equiv \operatorname{dim} \operatorname{Sec} X(\bmod 2)$ (i.e., $n+\varepsilon \equiv 0(\bmod 4))$ and $K_{X} \cong \mathcal{O}_{X}((-3 n+\varepsilon) / 4)$. In particular $X$ is a Fano manifold.

(3) If $\operatorname{dim} \operatorname{Sec} X=2 n-2$ (i.e., $\varepsilon=n-6)$, then $n$ is odd and $\left.c_{2}\left(\Omega_{X}\right)\right|_{\theta_{u}}=$ $(n+5)(n+1) / 4$.

(4) If $\operatorname{dim} \operatorname{Sec} X=2 n-3$ (i.e., $\varepsilon=n-8)$, then $n$ is even and

$$
\left.c_{2}\left(\Omega_{X}\right)\right|_{\theta_{u}}=(1 / 32)\left(9 n^{2}-(8+6 \varepsilon) n+32+8 \varepsilon+\varepsilon^{2}\right) c_{1}\left(\mathcal{O}_{\theta_{u}}(1)\right)^{2} .
$$


(5) If $\operatorname{dim} \operatorname{Sec} X \leq 2 n-4$ (i.e., $\varepsilon \leq n-10)$, then $n+1 \equiv \operatorname{dim} \operatorname{Sec} X(\bmod 4)$ (i.e., $n+\varepsilon \equiv 0(\bmod 8))$ and

$$
c_{2}\left(\Omega_{X}\right)=(1 / 32)\left(9 n^{2}-(8+6 \varepsilon) n+32+8 \varepsilon+\varepsilon^{2}\right) c_{1}\left(\mathcal{O}_{X}(1)\right)^{2} .
$$

Proof. First we have $a_{1}(m)=(m+2) / 2$ and $a_{2}(m)=\left(m^{2}+4 m+8\right) / 8$. Therefore $a_{1}((n+\varepsilon) / 2)=(n+4+\varepsilon) / 4$ and $a_{2}((n+\varepsilon) / 2)=\left(n^{2}+(8+2 \varepsilon) n+\right.$ $\left.32+8 \varepsilon+\varepsilon^{2}\right) / 32$. Let $h=c_{1}\left(\mathcal{O}_{\theta_{u}}(1)\right)$. Note that $c_{1}\left(\beta^{*} E\right)=\left.K_{X}\right|_{\theta_{u}}+(n+1) h$ and that $c_{2}\left(\beta^{*} E\right)=(1 / 2) n(n+1) h^{2}+\left.n K_{X}\right|_{\theta_{u}} \cdot h+\left.c_{2}\left(\Omega_{X}\right)\right|_{\theta_{u}}$. Hence it follows from Proposition 2.8 (1) that

$$
\left.K_{X}\right|_{\theta_{u}}=(1 / 4)(-3 n+\varepsilon) h
$$

and that

$$
\left.c_{2}\left(\Omega_{X}\right)\right|_{\theta_{u}}=(1 / 32)\left(9 n^{2}-(8+6 \varepsilon) n+32+8 \varepsilon+\varepsilon^{2}\right) h^{2} .
$$

If $\varepsilon=n-4$, then the above formula shows that $K_{X} \cdot \theta_{u}=-n-2$ since $h=2$ in this case. For the case $0 \leq \varepsilon \leq n-6$, we first observe that we can embed $X$ by the linear projection into $\mathbf{P}^{N^{\prime}}$ where $N^{\prime}=\operatorname{dim} \operatorname{Sec} X$. Then $\operatorname{dim} X-\operatorname{codim}\left(X, \mathbf{P}^{N^{\prime}}\right)=n-\left(N^{\prime}-n\right)=\operatorname{dim} \theta_{u}=(n-2-\varepsilon) / 2$. Thus if $\varepsilon \leq n-6$ then $\operatorname{dim} X-\operatorname{codim}\left(X, \mathbf{P}^{N^{\prime}}\right) \geq 2$, so that $\operatorname{Pic} \mathbf{P}^{N^{\prime}} \cong \operatorname{Pic} X$ by [O, Corollary 4.10]. Therefore we have $K_{X} \cong \mathcal{O}_{X}(t)$ for some integer $t$. Hence the above formula shows that $t=(-3 n+\varepsilon) / 4$ since Pic $\theta_{u}$ is torsion free. Thus we have $-3 n+\varepsilon \equiv 0(\bmod 4)$, that is, $n+\varepsilon \equiv 0(\bmod 4)$, and $K_{X} \cong \mathcal{O}_{X}((-3 n+\varepsilon) / 4)$.

If $\varepsilon=n-6$, then $\operatorname{dim} \theta_{u}=2$ and $h^{2}=2$. Thus the above formula gives the desired equality $\left.c_{2}\left(\Omega_{X}\right)\right|_{\theta_{u}}=(n+5)(n+1) / 4$.

For the case $\varepsilon \leq n-10$, we have $\operatorname{dim} X-\operatorname{codim}\left(X, \mathbf{P}^{N^{\prime}}\right)=(n-3) / 2 \geq 4$. Thus the natural maps $H^{i}\left(\mathbf{P}^{N^{\prime}}, \mathbf{Z}\right) \rightarrow H^{i}(X, \mathbf{Z})$ are isomorphisms for $i=$ $0,1, \cdots, 4$ (see $[\mathbf{L a}])$. It is well known that there is a theory of Chern classes with values in the cohomology ring $H^{*}(X, \mathbf{Z})$. It follows that $c_{2}\left(\Omega_{X}\right)=$ $t c_{1}\left(\mathcal{O}_{X}(1)\right)^{2}$ for some integer $t$. Note that $h^{2}$ is not a torsion element since $h^{\operatorname{dim} \theta_{u}}=\operatorname{deg} \theta_{u}=2$. Hence the above formula yields an equality $t=$ $\left(9 n^{2}-(8+6 \varepsilon) n+32+8 \varepsilon+\varepsilon^{2}\right) / 32$. Thus $0 \equiv 9 n^{2}-(8+6 \varepsilon) n+8 \varepsilon+\varepsilon^{2} \equiv 9(n+\varepsilon)^{2}$ $\left(\bmod 2^{5}\right)$ since $n+\varepsilon \equiv 0(\bmod 4)$. Therefore we have $n+\varepsilon \equiv 0\left(\bmod 2^{3}\right)$ and $c_{2}\left(\Omega_{X}\right)=(1 / 32)\left(9 n^{2}-(8+6 \varepsilon) n+32+8 \varepsilon+\varepsilon^{2}\right) c_{1}\left(\mathcal{O}_{X}(1)\right)^{2}$.

Remark 2.10. In the proposition above, only known examples of the case (5) are complete intersections of the $E_{6}$-manifold $E^{16} \subset \mathbf{P}^{26}$ and a codimension $m$ general linear subspace of $\mathbf{P}^{26}(m=0,1,2,3)$.

Proposition 2.11. Suppose that $\operatorname{dim} C_{u}=2 n+2-\operatorname{dim} \operatorname{Sec} X$ for a general point $u \in \operatorname{Sec} X$. Assume that $\varepsilon \leq n-6$. Let $m$ be an integer $\geq 2$, and $l$ an odd, positive integer such that $n+\varepsilon=2^{m} l$. Then $\varepsilon \geq 2^{m-1}(l-4)+4$. Moreover possible values of $n$ are

(1) a) 10,14 , or b) $2^{m}-2(m \geq 7), 2^{m} \cdot 3-2(m \geq 5)$ if $\varepsilon=2$; 
(2) a) 9,13 , or b) $21,2^{m}-3(m \geq 7), 2^{m} \cdot 3-3(m \geq 5)$ if $\varepsilon=3$;

(3) a) 12 , or b) $20,28,2^{m}-4(m \geq 7), 2^{m} \cdot 3-4(m \geq 5)$ if $\varepsilon=4$;

(4) a) 11 , or b) $19,27,2^{m}-5(m \geq 7), 2^{m} \cdot 3-5(m \geq 5)$ if $\varepsilon=5$.

Proof. Recall that $f_{2^{m}-2}\left(2^{m-1} \cdot l\right)$ is not an integer by Lemma $2.6(1)$. Thus Proposition $2.8(3)$ implies that an equality $(n+\varepsilon) / 2=2^{m-1} \cdot l$ is not compatible with an inequality $2^{m}-2 \leq(n-2-\varepsilon) / 4$. This means that $\varepsilon \geq 2^{m-1}(l-4)+4$ if $n+\varepsilon=2^{m} l$. Therefore $n=2^{m}-\varepsilon$ or $2^{m} \cdot 3-\varepsilon$ if $\varepsilon \leq 5$.

If $\varepsilon=2$, then $n \geq \varepsilon+6=8$. Moreover we have $n \geq 10$ since $n+2 \equiv 0$ $(\bmod 4)$ by Proposition $2.9(2)$. For $n=10$, there exists an example, which is the Grassmann manifold $G\left(1, \mathbf{P}^{6}\right)$ embedded in $\mathbf{P}^{20}$ via the Plücker embedding. If $n \geq \varepsilon+10=12$, then $n+2 \equiv 0(\bmod 8)$ by Proposition $2.9(5)$. A codimension 2 linear section of the $E_{6}$-variety is an example for the case $n=14$. If $n \geq 22$, then $n=2^{m}-2(m \geq 5)$ or $2^{m} \cdot 3-2(m \geq 3)$. Suppose that $n=2^{5}-2$. Then we have $f_{i}(16)+f_{i-1}(16) \geq 0$ for all $i(0 \leq i \leq 13)$ by Proposition $2.8(4)$. However this is a contradiction since $f_{12}(16)=-528$ and $f_{11}(16)=156$ by [T, p. 10, Table]. Similarly we get $n \neq 2^{6}-2$ since $f_{17}(32)+f_{16}(32)<0, n \neq 2^{3} \cdot 3-2$ since $f_{9}(12)+f_{8}(12)<0$, and $n \neq 2^{4} \cdot 3-2$ since $f_{17}(24)+f_{16}(24)<0$ by [T, p. 10, Table].

If $\varepsilon=3$, then $n \geq \varepsilon+6=9$. By Proposition 2.9 (2) we have $n+3 \equiv 0$ $(\bmod 4)$. For $n=9$, we have an example, that is, a smooth hyperplane section of $G\left(1, \mathbf{P}^{6}\right) \subset \mathbf{P}^{20}$. As above, a codimension 3 linear section of the $E_{6}$-variety is an example for the case $n=13$. If $n \geq 13$, then $n+3 \equiv 0$ $(\bmod 8)$ by Proposition $2.9(5)$. If $n \geq 21$, then $n=2^{m}-3(m \geq 5)$ or $2^{m} \cdot 3-3(m \geq 3)$. Since $f_{12}(16)+f_{11}(16)<0$, we know that $n \neq 29$. By the same argument, we know that $n \neq 61$ and that $n \neq 45$.

Finally for $\varepsilon=4$ and 5 we obtain the result by the same argument as above.

Remark 2.12. In the proposition above, no examples are known for the case b). Moreover we cannot eliminate the cases $(\varepsilon, n)=(3,21),(4,20)$, $(4,28),(5,19)$, and $(5,27)$ by Tango's argument unlike the cases that $\varepsilon=1$ or 2. Note also that if there exists a projective manifold $X \subset \mathbf{P}^{N}$ of $(\varepsilon, n)=$ $(3,21)$ then the secant defect $\delta=2 n+1-\operatorname{dim} \operatorname{Sec} X$ of this manifold is nine.

Now a proof of Theorem 0.2 follows immediately from Corollary 2.2, Proposition 2.9, and Proposition 2.11 if we take account of the case $\varepsilon \geq n-4$.

\section{The case that $\operatorname{dim} \operatorname{Sec} X=2 n-1$.}

In this section, let $X$ be an $n$-dimensional nondegenerate projective manifold in $\mathbf{P}^{N}$ with degenerate secant variety $\operatorname{Sec} X$ of dimension $2 n-1$, and let $L=\mathcal{O}_{X}(1)$. Then $\left|K_{X}+(n-1) L\right|$ is base point free by [Oh, Theorem 3.5]. 
Let $\phi: X \rightarrow \mathbf{P}\left(H^{0}\left(K_{X}+(n-1) L\right)\right)$ be the adjunction map, and let $\phi=s \circ r$ $\left(r: X \rightarrow Y, s: Y \rightarrow \mathbf{P}\left(H^{0}\left(K_{X}+(n-1) L\right)\right)\right)$ be the Stein factorization of $\phi$.

Proposition 3.1. If $n \geq 5$, then there are the following possibilities.

(1): $Y$ is a smooth projective surface, $(X, L) \cong(\mathbf{P}(\mathcal{E}), H(\mathcal{E}))$ over $Y$ for some vector bundle $\mathcal{E}$ of rank $n-1$ on $Y, K_{Y}+c_{1}(\mathcal{E})$ is very ample, and

$$
2 L^{n}+n K_{Y} c_{1}(\mathcal{E})+c_{1}(\mathcal{E})^{2}+\frac{\left(n^{2}-1\right)}{6}\left(K_{Y}^{2}-c_{2}\left(T_{Y}\right)\right)=0
$$

(2): $Y$ is smooth and $n$-dimensional, $r$ is the blowing-up at a finite point set of $Y, L=r^{*} M-\Sigma E_{i}\left(E_{i}\right.$ : exceptional divisors $)$ for some ample line bundle $M$ on $Y, K_{Y}+(n-1) M$ is very ample, and $K_{Y}+(n-2) M$ is nef.

Proof. If $n \geq 5$, then $\operatorname{dim} Y=2$ or $n$ by [Oh, Theorem 3.5]. If $\operatorname{dim} Y=2$, then we obtain (1) by [Oh, Theorem 3.5] except the assertion that $K_{Y}+c_{1}(\mathcal{E})$ is very ample. The assertion that $K_{Y}+c_{1}(\mathcal{E})$ is very ample follows from $[\mathbf{L}-\mathbf{M}$, Theorem $\mathrm{B}$ and Theorem $\mathrm{C}] \operatorname{since} \operatorname{rk} \mathcal{E}=n-1 \geq 4$. If $\operatorname{dim} Y=$ $n$, then we have (2) by [S-V, (0.3) and (2.1)] except the assertion that $K_{Y}+(n-2) M$ is nef. Finally this assertion follows from [Fb, (11.8)].

Proposition 3.2. Suppose that $n \geq 5$ and that the case (1) of Proposition 3.1 holds. Assume that $\operatorname{dim} C_{u}=2 n+2-\operatorname{dim} \operatorname{Sec} X=3$ for $a$ general point $u \in \operatorname{Sec} X$. Then $(Y, \mathcal{E}) \cong\left(\mathbf{P}^{2}, \mathcal{O}(1)^{\oplus(n-1)}\right)$, i.e., $(X, L) \cong$ $\left(\mathbf{P}^{2} \times \mathbf{P}^{n-2}, \mathcal{O}(1) \otimes \mathcal{O}(1)\right)$.

Proof. Since $\left.L\right|_{\theta_{u}}=2$ by Proposition 2.1, we have $\left.\left(K_{X}+(n-1) L\right)\right|_{\theta_{u}}=n-4$ by Proposition $2.9(1)$. Since $\left.\left(K_{X}+(n-1) L\right)\right|_{\theta_{u}}=n-4>0, \operatorname{dim} r\left(\theta_{u}\right)=1$ for a general point $u \in \operatorname{Sec} X$. Furthermore, since $\theta_{u}$ 's ( $u \in \operatorname{Sec} X$ : general) are algebraically equivalent, so are $r_{*}\left(\theta_{u}\right)$ 's $(u \in \operatorname{Sec} X$ : general) by [Fl, Proposition 10.3]. Moreover general two points $x, y$ on $X$ can be joined by $Q_{u}$, and so by its smooth hyperplane section $\theta_{u}$ for a general point $u \in \operatorname{Sec} X$. Hence there exist two general points $u, v$ on Sec $X$ such that $r\left(\theta_{u}\right) \cap r\left(\theta_{v}\right) \neq$ $\emptyset$ and $r\left(\theta_{u}\right) \neq r\left(\theta_{v}\right)$. Thus $r\left(\theta_{u}\right)^{2} \geq 1$ for a general point $u \in \operatorname{Sec} X$. Since $\left.\left(K_{X}+(n-1) L\right)\right|_{\theta_{u}}=n-4$, we have $\left.\left(K_{Y}+c_{1}(\mathcal{E})\right)\right|_{r\left(\theta_{u}\right)} \leq n-4$ for a general point $u \in \operatorname{Sec} X$. Therefore the Hodge index theorem implies $\left(K_{Y}+c_{1}(\mathcal{E})\right)^{2} r\left(\theta_{u}\right)^{2} \leq(n-4)^{2}$. Hence we have $\left(K_{Y}+c_{1}(\mathcal{E})\right)^{2} \leq(n-4)^{2}$.

Since $Y$ is rational by Corollary 2.2 and $\operatorname{dim} \operatorname{Sec} X=2 n-1, Y$ is $\mathbf{P}^{2}$ and $c_{1}(\mathcal{E}) \cong \mathcal{O}(n \pm 1)$ by [I-T, Proposition 4]. Because $\left(K_{Y}+c_{1}(\mathcal{E})\right)^{2} \leq(n-4)^{2}$, we have $c_{1}(\mathcal{E}) \cong \mathcal{O}(n-1)$. Hence $\mathcal{E}=\mathcal{O}(1)^{\oplus(n-1)}$ by the ampleness of $\mathcal{E}$.

Now we give a proof of Theorem 0.3 .

Proof of Theorem 0.3. By Proposition 3.1, $\operatorname{dim} Y=2$ or 6 . If $\operatorname{dim} Y=2$, then $(X, L) \cong\left(\mathbf{P}^{2} \times \mathbf{P}^{4}, \mathcal{O}(1) \otimes \mathcal{O}(1)\right)$ by Proposition 3.2. 
Suppose that $\operatorname{dim} Y=6$. Let $M$ be as in Proposition 3.1 (2). Since $n=6$, we have $2=\left.\left(K_{X}+5 L\right)\right|_{\theta_{u}}=\left.\left(K_{Y}+5 M\right)\right|_{r\left(\theta_{u}\right)}$. Thus $\left.\left(K_{Y}+4 M\right)\right|_{r\left(\theta_{u}\right)}=0$ because $\left.M\right|_{r\left(\theta_{u}\right)} \geq 2$ and $K_{Y}+4 M$ is nef. On the other hand, there exists a positive integer $m$ such that Bs $\left|m\left(K_{Y}+4 M\right)\right|=\emptyset$ by the Base Point Free Theorem ([K-M-M, Theorem 3-1-1]). Let $\varphi: Y \rightarrow Z$ be the morphism onto a normal projective variety $Z$ with connected fibers obtained from the Stein factorization of the morphism defined by $\left|m\left(K_{Y}+4 M\right)\right|$. Then there exists an ample divisor $A$ on $Z$ such that $K_{Y}+4 M \cong \varphi^{*} A$ by the Contraction Theorem ([K-M-M, Theorem 3-2-1]). Noting that general two points can be joined by $r\left(\theta_{u}\right)$ for a general point $u \in \operatorname{Sec} X$, we know that $Z$ is a point since $\left.\left(K_{Y}+4 M\right)\right|_{r\left(\theta_{u}\right)}=0$. Therefore $A=0$ and $K_{Y}+4 M=0$. Hence $Y$ is a Fano manifold, and for each point $y \in Y$ there is a rational curve $l$ on $Y$ such that $y \in l$ and $-\left.K_{Y}\right|_{l} \leq 7$ by [Mo]. Thus $\left.M\right|_{l}=1$. This implies that $r$ is an isomorphism because of the ampleness of $L$. Therefore $X$ is a 6 -dimensional Fano manifold of coindex 3 , and we have $h^{0}(L)=\operatorname{dim} X+g(X, L)-1$ where $g(X, L)$ is the sectional genus of the polarized manifold $(X, L)$ (see, for example, [Ko, Chap. V, 1.12.6 Exercise]). Furthermore $X$ satisfies the assumption (ES) in [Mu] since $L$ is very ample. We also have $h^{0}(L) \geq 13$ because $\operatorname{Sec} X \neq \mathbf{P}^{N}$ and $\operatorname{dim} \operatorname{Sec} X=11$. Hence $g(X, L) \geq 8$. Therefore $X \subset \mathbf{P}^{N}$ is one of a Segre variety $\mathbf{P}^{3} \times \mathbf{P}^{3} \subset \mathbf{P}^{15}$, a linear section of $G\left(1, \mathbf{P}^{5}\right) \subset \mathbf{P}^{14}$ by codimension 2 linear subspace of $\mathbf{P}^{14}$, or $\Sigma_{9} \subset \mathbf{P}^{13}$ by $\left[\mathbf{M u}\right.$, Theorem 2 and Theorem 7]. However $\operatorname{Sec} \Sigma_{9}=\mathbf{P}^{13}$ by $[\mathbf{K}]$, which contradicts the assumption that $\operatorname{dim} \operatorname{Sec} X=11$. On the other hand, the Segre manifold satisfies this assumption and the condition that $\operatorname{dim} C_{u}=3$, and the linear section of the Grassmann manifold satisfies the assumption that $\operatorname{dim} \operatorname{Sec} X=11$ by Proposition 1.4.

Remark 3.3. A linear section of the Grassmann manifold cut out by codimension 2 general linear subspace satisfies the condition that $\operatorname{dim} C_{u}=3$ by Proposition 1.7. However it is uncertain whether every smooth linear section satisfies the condition or not.

\section{The case that $\operatorname{dim} \operatorname{Sec} X=2 n$.}

In this section we investigate $n$-dimensional projective manifolds with degenerate secant varieties of dimension $2 n$. First of all, we state a couple of Lemmas needed later.

Lemma 4.1. Let $X \subseteq \mathbf{P}^{N}$ be an $n$-dimensional projective manifold. Then $\operatorname{dim} \operatorname{Sec} X \leq 2 n$ if and only if

$$
(\operatorname{deg} X)^{2}-\sum_{j=0}^{n}\left(\begin{array}{c}
2 n+1 \\
j
\end{array}\right) c_{1}\left(\mathcal{O}_{X}(1)\right)^{j} s_{n-j}\left(T_{X}\right) \cap[X]=0 .
$$

For a proof, see $[\mathbf{F},(1.5)$ and (1.7)] or $[\mathbf{H o}]$. 
Lemma 4.2. Let $X \subseteq \mathbf{P}^{N}$ be an $n$-dimensional projective manifold, $L=$ $\mathcal{O}_{X}(1)$, and assume that $(X, L) \cong\left(\mathbf{P}_{Y}(\mathcal{E}), H(\mathcal{E})\right)$ for some locally free sheaf $\mathcal{E}$ of rank $n-m+1$ on an $m$-dimensional projective manifold $Y$. Then

$$
\begin{aligned}
&\left(L^{n}\right)^{2}- \sum_{j=0}^{n}\left(\begin{array}{c}
2 n+1 \\
j
\end{array}\right) c_{1}(L)^{j} s_{n-j}\left(T_{X}\right) \cap[X] \\
&=\left(s_{m}(\check{\mathcal{E}})\right)^{2}-\sum_{j=0}^{n} \sum_{p=0}^{n-j} \sum_{l=0}^{p}\left(\begin{array}{c}
2 n+1 \\
j
\end{array}\right)\left(\begin{array}{c}
-n+m-l-1 \\
p-l
\end{array}\right) \\
&=\left\{\begin{array}{l}
c_{1}(\mathcal{E})^{2}-(2 n+1) c_{1}(\mathcal{E})-n(n+1)(g(Y)-1) \\
\left(L^{2}\right)^{2}-\left(n^{2}+n+1\right) L^{2} \\
-(1 / 6)(2 n+1)(n+1) n c_{1}(\mathcal{E}) c_{1}\left(K_{Y}\right) \\
-\left(\begin{array}{c}
n+2 \\
4
\end{array}\right)\left(c_{1}\left(K_{Y}\right)^{2}-c_{2}\left(T_{Y}\right)\right)-\left(\begin{array}{c}
n+1 \\
2
\end{array}\right) c_{1}(\mathcal{E})^{2}
\end{array}\right. \\
&
\end{aligned}
$$

If $Y=\mathbf{P}^{1}$, then $\operatorname{dim} \operatorname{Sec} X \leq 2 n$ if and only if $\mathcal{E} \cong \mathcal{O}(1)^{\oplus n}$ or $\mathcal{O}(1)^{\oplus(n-1)} \oplus$ $\mathcal{O}(2)$, and if $Y=\mathbf{P}^{1}$ and $\operatorname{dim} \operatorname{Sec} X \leq 2 n$, then $\operatorname{Sec} X=\mathbf{P}\left(H^{0}(L)\right)$.

Proof. We obtain these results by calculation and by Lemma 4.1.

We get the following lemma by calculation.

Lemma 4.3. Let $C$ be a smooth complete curve of genus $g$ and $\mathcal{E}$ a vector bundle of rank $n$ on $C$. Let $X$ be a smooth irreducible effective Cartier divisor of $\mathbf{P}(\mathcal{E})$ such that $\mathcal{O}_{\mathbf{P}(\mathcal{E})}(X) \cong H(\mathcal{E})^{\otimes 2} \otimes \pi^{*} M$ for some line bundle $M$ of degree $m$ on $C$, where $\pi: \mathbf{P}(\mathcal{E}) \rightarrow C$ is the projection. Let $L=H(\mathcal{E}) \otimes \mathcal{O}_{X}$ and $d=L^{n}$. Then

$$
d^{2}-\sum_{j=0}^{n}\left(\begin{array}{c}
2 n+1 \\
j
\end{array}\right) c_{1}(L)^{j} s_{n-j}\left(T_{X}\right) \cap[X]=d^{2}-4 n d-m-4 n^{2}(g-1) .
$$

In the rest of this section, let $X$ be an $n$-dimensional nondegenerate projective manifold in $\mathbf{P}^{N}$ with degenerate secant variety $\operatorname{Sec} X$ of dimension $2 n$, and let $L=\mathcal{O}_{X}(1)$.

Proposition 4.4. If $\operatorname{dim} C_{u}=2 n+2-\operatorname{dim} \operatorname{Sec} X=2$ for a general point $u \in \operatorname{Sec} X$, then we have $\operatorname{Bs}\left|K_{X}+(n-1) L\right|=\emptyset$ for all $n \geq 3$.

Proof. If $\mathrm{Bs}\left|K_{X}+(n-1) L\right| \neq \emptyset$, then $(X, L) \cong\left(\mathbf{P}_{C}(\mathcal{E}), H(\mathcal{E})\right)$ for some vector bundle $\mathcal{E}$ of rank $n$ on a smooth curve $C$ by $[\mathbf{S}-\mathbf{V},(0.1)]$ since $\operatorname{Sec} X \neq$ $\mathbf{P}^{N}$ and $n \geq 3$. Because $X$ is rationally connected by Proposition 2.2 , so is $C$, and hence $C=\mathbf{P}^{1}$. Therefore $\operatorname{Sec} X=\mathbf{P}\left(H^{0}(L)\right)$ by Lemma 4.2, which contradicts the hypothesis that $\operatorname{Sec} X \neq \mathbf{P}^{N}$. 
Remark 4.5. If $n=3$, then $K_{X}+2 L=0$ without the hypothesis that $\operatorname{dim} C_{u}=2$ for a general point $u \in \operatorname{Sec} X$ by $[\mathbf{F},(2.8)]$. However it is unsolved whether $\left|K_{X}+(n-1) L\right|$ is base point free or not for all $n \geq 4$ without the hypothesis that $\operatorname{dim} C_{u}=2$ for a general point $u \in \operatorname{Sec} X$.

In the following, we always assume that $\operatorname{dim} C_{u}=2$ for a general point $u \in \operatorname{Sec} X$ and that $n \geq 4$, and let $\phi: X \rightarrow \mathbf{P}\left(H^{0}\left(K_{X}+(n-1) L\right)\right)$ be the adjunction map, and let $\phi=s \circ r\left(r: X \rightarrow Y, s: Y \rightarrow \mathbf{P}\left(H^{0}\left(K_{X}+(n-1) L\right)\right)\right)$ be the Stein factorization of $\phi$.

Theorem 4.6. Under the assumptions above, there are the following possibilities.

(1): $Y$ is a smooth rational surface, $s$ is a closed immersion induced by $\left|K_{Y}+c_{1}(\mathcal{E})\right|,\left(K_{Y}+c_{1}(\mathcal{E})\right)^{2} \leq(n-3)^{2},(X, L) \cong\left(\mathbf{P}_{Y}(\mathcal{E}), H(\mathcal{E})\right)$ for some vector bundle of rank $n-1$ on $Y$, and

$$
\begin{aligned}
&\left(L^{2}\right)^{2}-\left(n^{2}+n+1\right) L^{2}-(1 / 6)(2 n+1)(n+1) n K_{Y} c_{1}(\mathcal{E}) \\
&-\left(\begin{array}{c}
n+2 \\
4
\end{array}\right)\left(K_{Y}^{2}-c_{2}\left(T_{Y}\right)\right)-\left(\begin{array}{c}
n+1 \\
2
\end{array}\right) c_{1}(\mathcal{E})^{2}=0 .
\end{aligned}
$$

Furthermore $\left(K_{Y}+c_{1}(\mathcal{E})\right)^{2} \geq 5$ unless $(Y, \mathcal{E}) \cong\left(\mathbf{P}^{2}, \mathcal{O}(1)^{\oplus l} \oplus \mathcal{O}(2)\right)$ or $\left(\mathbf{P}^{2}, \mathcal{O}(1)^{\oplus l-1} \oplus T_{\mathbf{P}^{2}}\right)$ where $l=2$ or 3 ;

(2): $Y$ is an $n$-dimensional rationally connected manifold, $r$ is the blowing-up of $Y$ at a finite point set, $L=r^{*} M-\Sigma E_{i}\left(E_{i}\right.$ : exceptional divisors) for some ample line bundle $M$ on $Y$, and $K_{Y}+(n-1) M$ is very ample. Moreover $\left.\left(K_{Y}+(n-2) M\right)\right|_{r\left(Q_{u}\right)} \leq n-5$ for a general point $u \in \operatorname{Sec} X, K_{Y}+(n-2) M$ is nef if $n \geq 5$, and $(Y, M) \cong\left(\mathbf{P}^{4}, \mathcal{O}(2)\right)$ if $n=4$.

Proof. First note that $Y$ is rationally connected because so is $X$ by Proposition 2.2. Since $\left.\left(K_{X}+(n-1) L\right)\right|_{Q_{u}}=n-3 \geq 1$ by Proposition 2.4, we have $\operatorname{dim} \phi(X) \geq 1$. Assume that $\operatorname{dim} Y=1$. Then $r$ is a quadric fibration over $Y$ by $[\mathbf{S}-\mathbf{V},(0.2)]$ and a contraction morphism of an extremal ray by $[\mathbf{B}-\mathbf{S}-\mathbf{W}$, Theorem (3.2.6)]. Therefore we can show, by the same argument as that in $[\mathbf{F b}$, p. $100,1.10-1.27]$, that there exist a locally free sheaf $\mathcal{E}$ of rank $n+1$ on $Y$ and a line bundle $M$ on $Y$ such that $X$ is a Cartier divisor of $\mathbf{P}(\mathcal{E})$, that $\mathcal{O}_{\mathbf{P}(\mathcal{E})}(X) \cong H(\mathcal{E})^{\otimes 2} \otimes \pi^{*} M$, and that $L \cong H(\mathcal{E}) \otimes \mathcal{O}_{X}$, where $\pi: \mathbf{P}(\mathcal{E}) \rightarrow Y$ is the projection and $r=\left.\pi\right|_{X}$. Since $Y$ is rationally connected, $Y$ is a smooth rational curve. Let $d=L^{n}, e=\operatorname{deg} c_{1}(\mathcal{E})$, and $m=\operatorname{deg} M$. Then we have $n-3=\left.\left(K_{X}+(n-1) L\right)\right|_{Q_{u}}=\left.\left(K_{\mathbf{P}(\mathcal{E})}+(n+1) H(\mathcal{E})+\pi^{*} M\right)\right|_{Q_{u}}=$ $\left.\pi^{*}\left(\mathcal{O}_{\mathbf{P}^{1}}(e+m-2)\right)\right|_{Q_{u}}$, and hence $e+m \leq n-1$. On the other hand, $\left(L^{n}\right)^{2}-\sum_{j=0}^{n}\left(\begin{array}{c}2 n+1 \\ j\end{array}\right) c_{1}(L)^{j} s_{n-j}\left(T_{X}\right) \cap[X]=(d-2 n)^{2}-m$ by Lemma 4.3, and therefore $\operatorname{dim} \operatorname{Sec} X=2 n$ implies that $(d-2 n)^{2}=m$. Let $m^{\prime}$ be a nonnegative integer such that $m=\left(m^{\prime}\right)^{2}$. Then we have $e=n-\left(m^{\prime}\left(m^{\prime} \mp 1\right) / 2\right)$ 
because $d=2 e+m$. It follows from $e+m \leq n-1$ that $m^{\prime 2} \pm m^{\prime}+2 \leq 0$, which is however a contradiction. Hence $\operatorname{dim} Y \geq 2$.

If $\operatorname{dim} Y=2$, then $Y$ is a smooth projective surface and $(X, L) \cong$ $\left(\mathbf{P}_{Y}(\mathcal{E}), H(\mathcal{E})\right)$ for some vector bundle of rank $n-1$ on $Y$ by $[\mathbf{S}-\mathbf{V},(0.2)]$. Furthermore $K_{Y}+c_{1}(\mathcal{E})$ is very ample by [L-M, Theorem B and Theorem C] because $H(\mathcal{E})$ is very ample, so that $s$ is a closed immersion. Note also that $Y$ is rational since $\operatorname{dim} Y=2$. For general three points $x, y, z \in X$, there exist two points $u, v \in \operatorname{Sec} X$ such that $x, y \in Q_{u}$ and $y, z \in Q_{v}$ and $u, v$ are in general position. Since $Q_{u}$ and $Q_{v}$ are algebraically equivalent, so is $r_{*}\left(Q_{u}\right)$ and $r_{*}\left(Q_{v}\right)$. Since $r(y) \in r\left(Q_{u}\right) \cap r\left(Q_{v}\right)$ and $r\left(Q_{u}\right) \neq r\left(Q_{v}\right)$, we get $r\left(Q_{u}\right)^{2} \geq 1$. We also have $\left(K_{Y}+c_{1}(\mathcal{E})\right)^{2} r\left(Q_{u}\right)^{2} \leq\left(\left.\left(K_{Y}+c_{1}(\mathcal{E})\right)\right|_{r\left(Q_{u}\right)}\right)^{2} \leq(n-3)^{2}$ by the Hodge index theorem. Therefore $\left(K_{Y}+c_{1}(\mathcal{E})\right)^{2} \leq(n-3)^{2}$. If $\left(K_{Y}+c_{1}(\mathcal{E})\right)^{2}=1$, then $Y=\mathbf{P}^{2}$ and $c_{1}(\mathcal{E}) \cong \mathcal{O}(4)$. If the rank of $\mathcal{E}$ is 3 , then $\mathcal{E} \cong \mathcal{O}(1)^{\oplus 2} \oplus \mathcal{O}(2)$ or $\mathcal{O}(1) \oplus T_{\mathbf{P}^{2}}$ by $[\mathbf{E} 1]$. In this case, $\operatorname{dim} \operatorname{Sec} X=8$ and $h^{0}(L) \geq 11$. The condition that $\operatorname{dim} C_{u}=2$ is also satisfied. If the rank of $\mathcal{E}$ is 4 , then $\mathcal{E} \cong \mathcal{O}(1)^{\oplus 4}$. Hence $(X, L) \cong\left(\mathbf{P}^{2} \times \mathbf{P}^{3}, \mathcal{O}(1) \otimes \mathcal{O}(1)\right)$, which, however, does not satisfy the condition that $\operatorname{dim} \operatorname{Sec} X=10$. If $\left(K_{Y}+c_{1}(\mathcal{E})\right)^{2} \geq 2$, then $\operatorname{rk} \mathcal{E} \geq 4$ since $(n-3)^{2} \geq 2$. If $\left(K_{Y}+c_{1}(\mathcal{E})\right)^{2}=2$, then $Y=\mathbf{P}^{1} \times \mathbf{P}^{1}$ and $c_{1}(\mathcal{E}) \cong \mathcal{O}(3) \otimes \mathcal{O}(3)$. This contradicts the ampleness of $\mathcal{E}$. If $\left(K_{Y}+c_{1}(\mathcal{E})\right)^{2}=3$, then $Y$ is either a cubic surface in $\mathbf{P}^{3}$ or $\mathbf{P}\left(\mathcal{O}_{\mathbf{P}^{1}}(1) \otimes \mathcal{O}_{\mathbf{P}^{1}}(2)\right)$ by [*]. If $Y$ is cubic, then $\left.c_{1}(\mathcal{E})\right|_{l}=2$ for every $l$, one of the 27 lines on $Y$, which is a contradiction. For the scroll we have $\left.c_{1}(\mathcal{E})\right|_{f}=3$ where $f$ is any fiber of the scroll, and this also contradicts the ampleness of $\mathcal{E}$. Suppose that $\left(K_{Y}+c_{1}(\mathcal{E})\right)^{2}=4$. Then $Y$ is either a del Pezzo surface of degree 4 , a scroll $\mathbf{P}\left(\mathcal{O}_{\mathbf{P}^{1}}(1) \oplus \mathcal{O}_{\mathbf{P}^{1}}(3)\right)$, a scroll $\mathbf{P}\left(\mathcal{O}_{\mathbf{P}^{1}}(2) \oplus \mathcal{O}_{\mathbf{P}^{1}}(2)\right)$, or a Veronese surface $\mathbf{P}^{2} \subset \mathbf{P}^{5}$ by $[\mathbf{S}]$ since $\kappa(Y)=-\infty$. If $Y$ is a del Pezzo surface, then $\left.c_{1}(\mathcal{E})\right|_{l}=2$ for any exceptional divisor $l$ of $Y$, which is a contradiction. For the scrolls we have $\left.c_{1}(\mathcal{E})\right|_{f}=3$ where $f$ is any fiber of the projection $Y \rightarrow \mathbf{P}^{1}$, and this is also a contradiction. If $Y$ is a Veronese surface, we obtain $c_{1}(\mathcal{E}) \cong \mathcal{O}(5)$. If $\mathcal{E}$ is an ample vector bundle of rank 4 , we have $\mathcal{E} \cong \mathcal{O}(1)^{\oplus 3} \oplus \mathcal{O}(2)$ or $\mathcal{O}(1)^{\oplus 2} \oplus T_{\mathbf{P}^{2}}$ by [E2, Theorem 5.1]. For both bundles, we have $\operatorname{dim} \operatorname{Sec} X=10$ and $h^{0}(L) \geq 14$. The condition that $\operatorname{dim} C_{u}=2$ is also satisfied. If the rank of $\mathcal{E}$ is 5 , then $\mathcal{E} \cong \mathcal{O}(1)^{\oplus 5}$. Hence $(X, L) \cong\left(\mathbf{P}^{2} \times \mathbf{P}^{4}, \mathcal{O}(1) \otimes \mathcal{O}(1)\right)$, which however does not satisfy the condition that $\operatorname{dim} \operatorname{Sec} X=12$. The rest of the assertion in the case $\operatorname{dim} Y=2$ follows from Lemma 4.2.

If $\operatorname{dim} Y>2$, then $\operatorname{dim} Y=n$ by $[\mathbf{S}-\mathbf{V},(0.2)]$. We also know that $Y$ is smooth, that $r$ is the blowing-up of $Y$ at a finite point set, and that $L=r^{*} M-\Sigma E_{i}\left(E_{i}\right.$ : exceptional divisors) for some ample line bundle $M$ on $Y$ by $[\mathbf{S}-\mathbf{V},(0.3)]$. Moreover $K_{Y}+(n-1) M$ is very ample by $[\mathbf{S}-\mathbf{V}$, Theorem (2.1)]. Since $n-3=\left.\left(K_{X}+(n-1) L\right)\right|_{Q_{u}}=\left.\left(K_{Y}+(n-1) M\right)\right|_{r\left(Q_{u}\right)}$ and $\left.M\right|_{r\left(Q_{u}\right)} \geq\left. L\right|_{Q_{u}}=2$, we obtain $\left.\left(K_{Y}+(n-2) M\right)\right|_{r\left(Q_{u}\right)} \leq n-5$. If $n \geq 5$, then we know that $K_{Y}+(n-2) M$ is nef by $[\mathbf{F b},(11.8)]$, taking account of 
the fact that $K_{Y}+(n-1) M$ is ample. If $n=4$, then $\left.\left(K_{Y}+2 M\right)\right|_{r\left(Q_{u}\right)} \leq-1$ and hence $K_{Y}+2 M$ is not nef. Therefore $(Y, M) \cong\left(\mathbf{P}^{4}, \mathcal{O}(2)\right)$ by $[\mathbf{F b}$, (11.8)] because $K_{Y}+3 M$ is ample.

Now we give a proof of Theorem 0.4 .

Proof of Theorem 0.4. Suppose first that $n=4$. Then Theorem 4.6 implies that $\operatorname{dim} Y=2$ or 4 . If $\operatorname{dim} Y=2$, then $(X, L)$ is isomorphic to $\left(\mathbf{P}_{\mathbf{P}^{2}}(\mathcal{E}), H(\mathcal{E})\right)$, where $\mathcal{E}=\mathcal{O}(1)^{\oplus 2} \oplus \mathcal{O}(2)$ or $\mathcal{O}(1) \oplus T_{\mathbf{P}^{2}}$ by Theorem 4.6 (1). If $\operatorname{dim} Y=4$, then $(Y, M) \cong\left(\mathbf{P}^{4}, \mathcal{O}(2)\right)$ by Theorem $4.6(2)$. If $r$ is an isomorphism, then $(X, L) \cong\left(\mathbf{P}^{4}, \mathcal{O}(2)\right)$. If $r$ is not an isomorphism, then $(X, L) \cong\left(\mathbf{P}_{\mathbf{P}^{3}}(\mathcal{O}(1) \oplus \mathcal{O}(2)), H(\mathcal{O}(1) \oplus \mathcal{O}(2))\right)$. These polarized manifolds satisfy the assumptions that $\operatorname{dim} \operatorname{Sec} X=8$ and that $\operatorname{dim} C_{u}=2$.

Suppose in the following that $n=5$. Then Theorem 4.6 shows that $\operatorname{dim} Y=2$ or 5 . If $\operatorname{dim} Y=2$, then $\left(X, \mathcal{O}_{X}(1)\right) \cong\left(\mathbf{P}_{\mathbf{P}^{2}}(\mathcal{E}), H(\mathcal{E})\right)$, where $\mathcal{E}=\mathcal{O}(1)^{\oplus 3} \oplus \mathcal{O}(2)$ or $\mathcal{O}(1)^{\oplus 2} \oplus T_{\mathbf{P}^{2}}$ by Theorem 4.6 (1). These two polarized manifolds satisfy the hypotheses that $\operatorname{dim} \operatorname{Sec} X=10$ and that $\operatorname{dim} C_{u}=2$.

Let us consider the case (2) of Theorem 4.6. Now we know that $K_{Y}+3 M$ is nef and therefore $\left.\left(K_{Y}+3 M\right)\right|_{r\left(Q_{u}\right)}=0$. Since general two points can be joined by $r\left(Q_{u}\right)$, this implies that $K_{Y}+3 M=0$ by [K-M-M, Theorem 31-1 and Theorem 3-2-1]. If $M$ is not the fundamental line bundle, then $(Y, M) \cong\left(\mathbf{P}^{5}, \mathcal{O}(2)\right)$. If $r$ is an isomorphism, then $(X, L) \cong\left(\mathbf{P}^{5}, \mathcal{O}(2)\right)$. If $r$ is not an isomorphism, then $(X, L) \cong\left(\mathbf{P}_{\mathbf{P}^{4}}(\mathcal{O}(1) \oplus \mathcal{O}(2)), H(\mathcal{O}(1) \oplus \mathcal{O}(2))\right)$. These two polarized manifolds satisfy the hypotheses that $\operatorname{dim} \operatorname{Sec} X=10$ and that $\operatorname{dim} C_{u}=2$.

Assume that $M$ is the fundamental line bundle of $Y$. Then $Y$ is a Fano manifold of coindex 3 and $M$ is very ample because $K_{Y}+4 M$ is very ample, so that $(Y, M)$ satisfies the hypothesis (ES) of $\left[\mathbf{M u}\right.$. If $B_{2}(Y) \geq 2$, then $(Y, M)$ is one of $\left(\mathbf{P}^{2} \times Q^{3}, \mathcal{O}(1) \otimes \mathcal{O}(1)\right),\left(\mathbf{P}\left(T_{\mathbf{P}^{3}}\right), H\left(T_{\mathbf{P}^{3}}\right)\right)$, or $\left(\mathbf{P}_{\mathbf{P}^{3}}\left(\mathcal{O}(1)^{\oplus 2} \oplus\right.\right.$ $\left.\mathcal{O}(2)), H\left(\mathcal{O}(1)^{\oplus 2} \oplus \mathcal{O}(2)\right)\right)$ by $[\mathbf{M u}$, Theorem 7]. Thus for every point $y \in Y$ there exists a line passing through $y$, which implies that $r$ is an isomorphism by the ampleness of $L$. Since the secant variety of the manifold $\left(\mathbf{P}^{2} \times\right.$ $\left.Q^{3}, \mathcal{O}(1) \otimes \mathcal{O}(1)\right)$ is 11-dimensional by [Z, Chap. 3, Theorem 1.6], $(X, L)$ is either $\left(\mathbf{P}\left(T_{\mathbf{P}^{3}}\right), H\left(T_{\mathbf{P}^{3}}\right)\right)$ or $\left(\mathbf{P}_{\mathbf{P}^{3}}\left(\mathcal{O}(1)^{\oplus 2} \oplus \mathcal{O}(2)\right), H\left(\mathcal{O}(1)^{\oplus 2} \oplus \mathcal{O}(2)\right)\right)$. These polarized manifolds satisfy the hypothesis that $\operatorname{dim} C_{u}=2$ and the condition that $\operatorname{dim} \operatorname{Sec} X=10$.

Next let us consider the case that $B_{2}(Y)=1$. Recall that $h^{0}(M)=$ $\operatorname{dim} Y+g(Y, M)-1$ for a pair of a Fano manifold $Y$ of coindex 3 and the fundamental line bundle $M$ on $Y$, where $g(Y, M)$ denotes the sectional genus of the polarized manifold $(Y, M)$ (see, for example, [Ko, Chap. V, 1.12.6 Exercise]). Hence $g(Y, M)+4=h^{0}(M) \geq h^{0}(L) \geq \operatorname{dim} \operatorname{Sec} X+2=12$.

Suppose that $r$ is an isomorphism. Then we get $g(X, L) \geq 8$. Thus $X \subset$ $\mathbf{P}^{N}$ is either a complete intersection of $G\left(1, \mathbf{P}^{5}\right) \subset \mathbf{P}^{14}$ and a codimension 
3 linear subspace of $\mathbf{P}^{14}$ or the $G_{2}$ adjoint manifold $\Sigma_{10} \subset \mathbf{P}^{13}$ by $[\mathbf{M u}$, Theorem 2], because Sec $\Sigma_{9}=\mathbf{P}^{13}$ by $[\mathbf{K}]$ and therefore the dimension of the secant variety of a general hyperplane section of $\Sigma_{9}$ is 11 by Proposition 1.5 and all smooth hyperplane sections of $\Sigma_{9}$ are isomorphic. A smooth complete intersection of $G\left(1, \mathbf{P}^{5}\right) \subset \mathbf{P}^{14}$ and a codimension 3 linear subspace of $\mathbf{P}^{14}$ satisfies the condition that $\operatorname{dim} \operatorname{Sec} X=10$ by Proposition 1.4. The $G_{2}$ adjoint manifold $\Sigma_{10} \subset \mathbf{P}^{13}$ satisfies the assumptions that $\operatorname{dim} \operatorname{Sec} X=10$ and that $\operatorname{dim} C_{u}=2$ by $[\mathbf{K}-\mathbf{O}-\mathbf{Y}]$.

Suppose that $r$ is not an isomorphism. Then $h^{0}(M) \geq h^{0}(L)+1$ so that $g(Y, M) \geq 9$. Therefore $Y$ is either $\Sigma_{10} \subset \mathbf{P}^{13}$ or a hyperplane section of $\Sigma_{9} \subset \mathbf{P}^{13}$. For each point $y \in \Sigma_{9}, \Sigma_{9}$ contains a rational curve $C$ passing through $y$ such that $-\left.K\right|_{C} \leq 7$ by [Mo]. Since the index of the Fano manifold $\Sigma_{9}$ is four, we have $-\left.K\right|_{C}=4$, and hence $C$ is a line in $\Sigma_{9}$ with respect to the very ample line bundle $M$ on $\Sigma_{9}$. Let $f: \mathbf{P}^{1}=C \hookrightarrow \Sigma_{9}$ be the inclusion of $C$ and let $f(0)=y$. Denote by $\iota$ the restriction of $f$ to $\{0\}$. Then $\operatorname{dim}_{[f]} \operatorname{Hom}\left(\mathbf{P}^{1}, \Sigma_{9} ; \iota\right) \geq-\left.K\right|_{C}=4$. On the other hand, we have $\operatorname{dim} \operatorname{Aut}\left(\mathbf{P}^{1} ; 0\right)=2$. Thus $\Sigma_{9}$ contains a closed cone of dimension $\geq 3$ with vertex $y$. A hyperplane section $Y$ of $\Sigma_{9}$ therefore contains a line passing through $y$ for each point $y \in Y$. This contradicts the ampleness of $L$. For the $G_{2}$ adjoint variety $\Sigma_{10} \subset \mathbf{P}^{13}$, it follows from $[\mathbf{K o}$, Chap. V, Theorem 1.15] that there exists a line $C$ (i.e., $\left.M\right|_{C}=1$ ) on $\Sigma_{10}$. Hence for every point $y \in \Sigma_{10}$ there exists a line passing through $y$ on $\Sigma_{10}$, which contradicts the ampleness of $L$.

Remark 4.7. A linear section of the Grassmann manifold cut out by codimension 3 general linear subspace satisfies the condition that $\operatorname{dim} C_{u}=2$ by Proposition 1.7. However it is uncertain whether every smooth linear section satisfies the condition or not.

\section{References}

[B-S-W] M.C. Beltrametti, A.J. Sommese and J.A. Wiśniewski, Results on varieties with many lines and their applications to adjunction theory, in Lecture Notes in Math., 1507, (eds. K. Hulek et al.), Springer-Verlag, Berlin, (1992), 16-38.

[E1] G. Elencwajg, Les fibrés uniformes de rang 3 sur $\mathbf{P}_{2}(\mathbf{C})$ sont homogènes, Math. Ann., 231 (1978), 217-227.

[E2] G. Elencwajg, Fibrés uniformes de rang élevé sur $\mathbf{P}_{2}$, Ann. Inst. Fourier (Grenoble), 31(4) (1981), 89-114.

[F] T. Fujita, Projective threefolds with small secant varieties, Sci. Papers Coll. Gen. Educ., Univ. Tokyo, 32(1) (1982), 33-46.

[Fb] T. Fujita, Classification theory of polarized varieties, London Math. Soc. Lecture Note Ser., 155, Cambridge University Press, Cambridge, 1990.

[F-R] T. Fujita and J. Roberts, Varieties with small secant varieties: The extremal case, Amer. J. Math., 103 (1981), 953-976. 
[Fl] W. Fulton, Intersection theory, Ergeb. Math. Grenzgeb., (3), Folge Band 2, Springer-Verlag, Berlin, 1984.

[H] R. Hartshorne, Algebraic geometry, Graduate Texts in Math., 52, SpringerVerlag, Berlin, 1983.

[Ho] A. Holme, Embedding obstruction for smooth projective varieties, I, in Advances in Math. Supplementary Studies, 5 (ed. Gian-Carlo Rota), Academic Press, Inc., (1979), 39-67.

[I-T] P. Ionescu and M. Toma, Boundedness for some special families of embedded manifolds, in Contemp. Math., 162, Amer. Math. Soc., (1994), 215-225.

[K] H. Kaji, Homogeneous projective varieties with degenerate secants, to appear in Trans. Amer. Math. Soc.

[K-O-Y] H. Kaji, M. Ohno and O. Yasukura, Adjoint varieties and their secant varieties, to appear in Indag. Math. (N.S.)

[K-M-M] Y. Kawamata, K. Matsuda and K. Matsuki, Introduction to the mimimal model problem, in Adv. Stud. Pure Math., 10 (ed. T. Oda), Kinokuniya, Tokyo, (1987), 283-360.

[Ko] J. Kollár, Rational curves on algebraic varieties, Ergeb. Math. Grenzgeb. (3), Folge Band 32, Springer-Verlag, Berlin, 1996.

[Ko-Mi-Mo] J. Kollár, Y. Miyaoka and S. Mori, Rationally connected varieties, J. Algebraic Geom., 1 (1992), 429-448.

[L] J.M. Landsberg, On degenerate secant and tangential varieties and local differential geometry, Duke Math. J., 85(3) (1996), 605-634.

[L-M] A. Lanteri and H. Maeda, Adjoint bundles of ample and spanned vector bundles on algebraic surfaces, J. Reine Angew. Math., 433 (1992), 181-199.

[La] M.E. Larsen, On the topology of complex projective manifolds, Invent. Math., 19 (1973), 251-260.

$[\mathrm{L}-\mathrm{V}] \quad \mathrm{R}$. Lazarsfeld and A. Van de Ven, Topics in the geometry of projective space, recent work of F.L. Zak, DMV Sem. Band 4, Birkhäuser, Basel, 1984.

[M-M] Y. Miyaoka and S. Mori, A numerical criterion for uniruledness, Ann. of Math., 124(2) (1986), 65-69.

[Mo] S. Mori, Projective manifolds with ample tangent bundles, Ann. of Math., 110(2) (1979), 593-606.

[Mu S. Mukai, Biregular classification of Fano 3-folds and Fano manifolds of coindex 3, Proc. Nat. Acad. Sci. U.S.A., 86 (1989), 3000-3002.

[O] A. Ogus, On the formal neighborhood of a subvariety of projective space, Amer. J. Math., 97 (1975), 1085-1107.

[Oh] M. Ohno, On odd dimensional projective manifolds with smallest secant varieties, Math. Z., 226 (1997), 483-498.

[S-V] A.J. Sommese and A. Van de Ven, On the adjunction mapping, Math. Ann., 278 (1987), 593-603.

[S] H.P.F. Swinnerton-Dyer, An enumeration of all varieties of degree 4, Amer. J. Math., 95 (1973), 403-418.

[T] H. Tango, Remark on varieties with small secant varieties, Bull. Kyoto Univ. Ed. Ser. B, 60 (1982), 1-10. 
[Z] F.L. Zak, Tangents and secants of algebraic varieties, Transl. Math. Monographs, 127, Amer. Math. Soc., 1993.

[*] $\quad$ ***, Correspondence, Amer. J. Math., 79 (1957), 951-952.

Received February 3, 1997 and revised November 18, 1997. Partially supported by Waseda University Grant for Special Research Projects, 96A-136.

WASEDA UNVERSITY

TOKYO, 169

JAPAN

E-mail address: mohno@mn.waseda.ac.jp 